\title{
EFFECTS OF ELECTROPHORETICALLY DEPOSITED CARBON NANOFIBERS ON THE INTERFACE OF SINGLE CARBON FIBERS EMBEDDED IN EPOXY MATRIX
}

\author{
A Thesis by \\ Joseph Daniel Schaefer \\ Bachelor of Science, University of Nebraska-Lincoln, USA, 2008
}

Submitted to the Department of Mechanical Engineering and the faculty of the Graduate School of

Wichita State University

in partial fulfillment of

the requirements for the degree of

Master of Science

May 2011 
(C) Copyright 2011 by Joseph Daniel Schaefer

All Rights Reserved 


\section{EFFECTS OF ELECTROPHORETICALLY DEPOSITED CARBON NANOFIBERS ON THE INTERFACE OF SINGLE CARBON FIBERS EMBEDDED IN EPOXY MATRIX}

The following faculty members have examined the final copy of this thesis for form and content, and recommend that it be accepted in partial fulfillment of the requirement for the degree of Master of Science with a major in Mechanical Engineering.

Bob Minaie, Committee Chair

Walter Horn, Committee Member

Ramazan Asmatulu, Committee Member 


\section{DEDICATION}

To my wife Kelly who continually challenges me to exceed my expectations, to my sister Anne who shows me new horizons, and to my parents for their patience, support, and foresight. 


\section{ACKNOWLEDGEMENTS}

This work would not be complete without expressing my gratitude to everyone who helped and supported my learning over the past two years. While there are too many of you to mention by name, please understand that this work would not be possible without you!

Specifically, I am indebted to my advisor, Dr. Bob Minaie, who gave me the independence, trust, and resources to direct this research. I would also like to thank Dr. Walter Horn and Dr. Ramazan Asmatulu for serving on my thesis committee. To my colleagues, Alejandro Rodriguez, Mauricio Guzman, and Chee Sern Lim: Thank you for sharing your

expertise, support, and enthusiasm. Working with you has been a privilege, and the success of this research is directly because of you. I am excited to follow your future successes!

I also wish to thank my other colleagues in the lab. Thank you for your collaboration, friendship, and shared experiences. Special thanks are due to Ronald Joven, Hoda Koushyar, Behrouz Tavakol, Mostafa Sabzevari, and Tien Vo for tolerating the many discussions held by my group in your workspace.

To my wife, Kelly, I express the grateful appreciation one can only have for a true friend. Your drive, motivation, and achievements challenge me to reach my goals, and your support and patience make it possible.

Financial support by the Office of Naval Research and the National Aeronautics and Space Administration is gratefully acknowledged. 


\begin{abstract}
In this work, electrophoretic deposition (EPD) was used to deposit carboxylic acidfunctionalized carbon nanofibers (O-CNFs) and amine-functionalized carbon nanofibers (ACNFs) on the surface of single carbon fibers. Using the fiber fragmentation technique, the stress transfer at the single fiber interface was characterized by determining the interfacial shear strength (IFSS) for different fiber surface treatments. For the O-CNF investigation, samples for sized, unsized, O-CNF deposited sized, and O-CNF deposited unsized carbon fibers were tested. The A-CNF investigation was completed for sized carbon fibers acting as the anode during EPD for single and double concentrations of CNFs in water. Additionally, results for fibers acting as both the anode and cathode during a two-stage A-CNF deposition process are provided. Finally, the effects of EPD were investigated by testing fibers acting as the cathode or anode in water without the presence of O-CNFs or A-CNFs. Weibull analyses of single fiber tensile failures were performed to account for scale effects along the fiber length and support IFSS estimation. This research was aimed at obtaining a fundamental understanding of how functionalized CNF addition, EPD electric field setup, and fiber sizing affected IFSS and fiber surface morphology. Additionally, the processing effects on single fiber tensile strength were determined.

It was shown that removing the sizing decreased the IFSS by $27 \%$. One-stage addition of O-CNFs to the unsized interface increased the IFSS by $15 \%$ over the base sized fiber and $56 \%$ compared to the unsized fibers. One-stage cathodic deposition of O-CNFs on the single fiber surface led to the greatest IFSS increase of 215\%. This IFSS increase is attributed to enhancement of surface roughness and surface area created by addition of the O-CNFs to the carbon fiber surface.
\end{abstract}




\section{TABLE OF CONTENTS}

Chapter

Page

1. INTRODUCTION 1

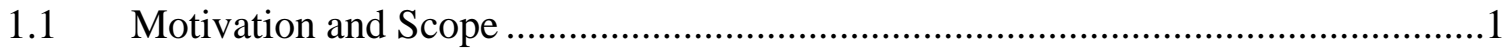

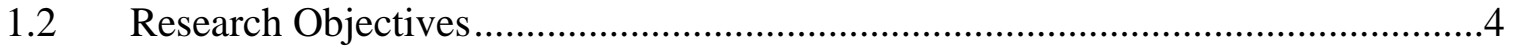

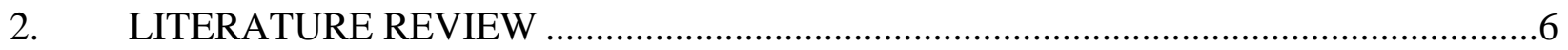

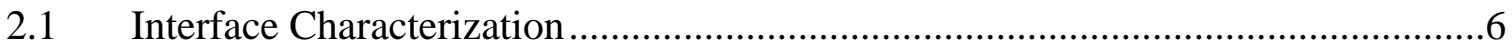

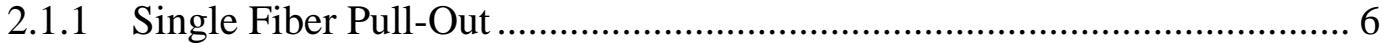

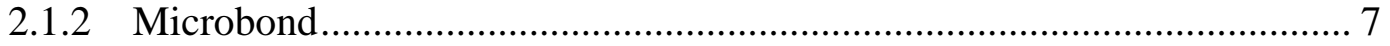

2.1.3 Single Fiber Fragmentation.................................................................... 9

2.2 Morphology and Properties of Carbon Nanotubes and Carbon Nanofibers ..........10

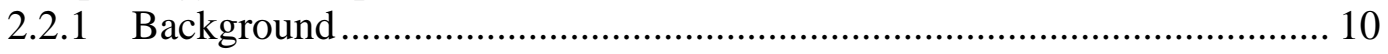

2.2.2 Carbon Nanotube Morphology ......................................................... 12

2.2.3 Carbon Nanofiber Morphology, Production, and Properties .................... 15

2.2.4 Properties of Carbon Nanotubes and Carbon Nanofibers......................... 18

2.3 Applications in Advanced Composites ............................................................19

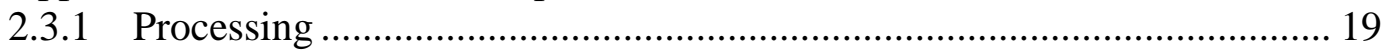

2.3.2 Properties of Nano-Reinforced Composites ......................................... 23

2.3.2.1 Polymer Nanocomposites ....................................................... 23

2.3.2.2 Nano-Reinforced Fabric Polymer Composites ......................... 24

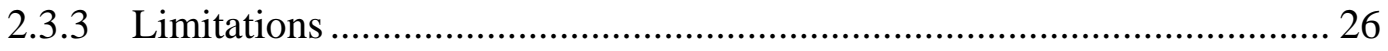

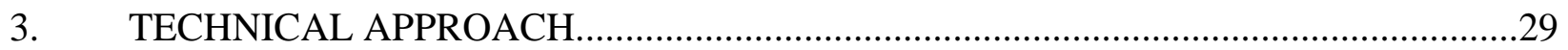

3.1 Materials and Experimental Methods …......................................................29

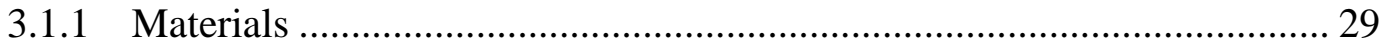

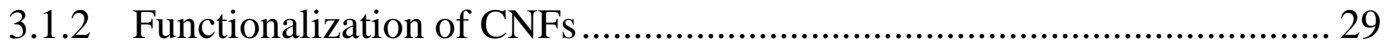

3.1.2.1 Carboxylic-Functionalization ................................................ 30

3.1.2.2 Amine-Functionalization ........................................................ 30

3.1.3 Electrophoretic Deposition of O-CNFs onto Single Carbon Fibers ......... 30

3.1.4 Electrophoretic Deposition of A-CNFs onto Single Carbon Fibers ......... 32

3.1.5 Tensile Strength Testing of Single Carbon Fibers ................................... 34

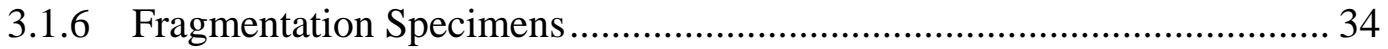

3.1.7 Single Fiber Fragmentation Test........................................................... 34

3.1.8 Weibull Analysis of Single Fiber Tensile Failures ................................ 36

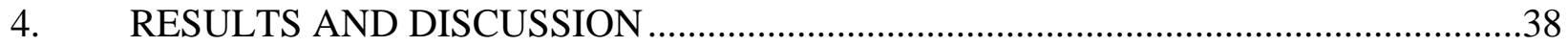

4.1 Effects of Electrophoretically Deposited O-CNFs on the Interface of Single Carbon Fibers Embedded in Epoxy Matrix 


\section{TABLE OF CONTENTS (continued)}

Chapter

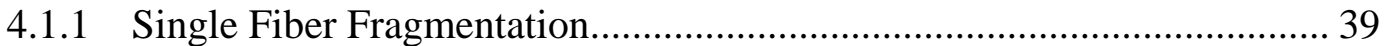

4.1.2 Interfacial Shear Strength ............................................................... 42

4.1.3 Surface Characteristics and Break Behavior......................................... 44

4.2 Effects of Electrophoretically Deposited A-CNFs on the Interface of Single Carbon Fibers Embedded in Epoxy Matrix .......................................................52

4.2.1 Tensile Strength and Weibull Parameters............................................ 52

4.2.2 Interfacial Shear Strength .............................................................. 53

4.2.3 Fiber Surface Characterization ......................................................... 59

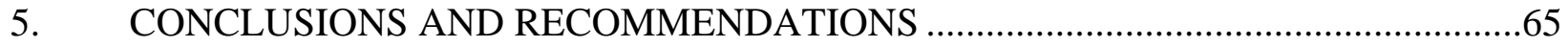

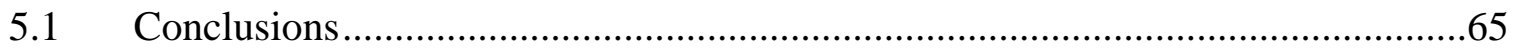

5.2 Recommendations for Further Studies.................................................67

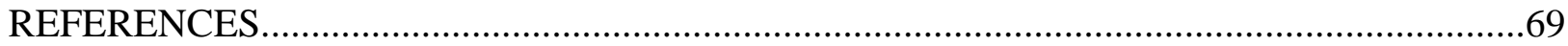




\section{LIST OF TABLES}

Table Page

1. Properties of VCGNFs, SWCNTs, and MWCNTs.................................................. 18

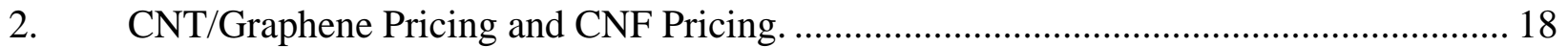

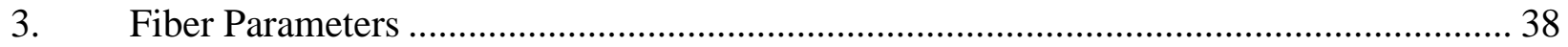

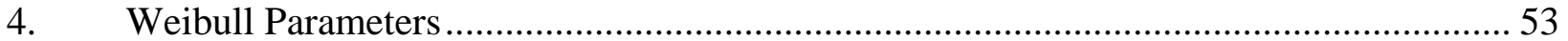




\section{LIST OF FIGURES}

Figure

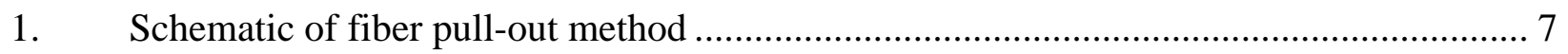

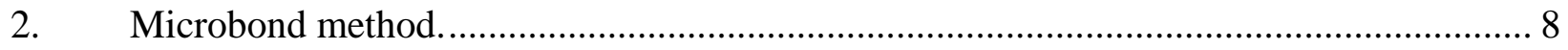

3. (a) HRTEM image of two crossing SWNTs coated with amorphous carbon; (b) Higher magnification of (a); (c) HRTEM image showing a MWNT upper region together with a

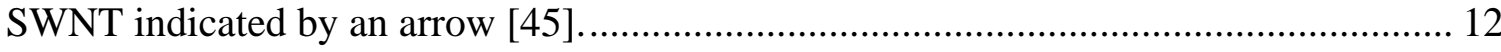

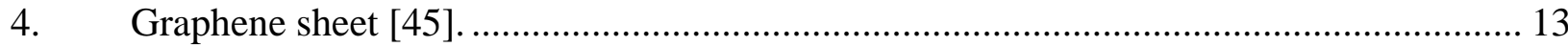

5. Molecular models of SWNTs exhibiting different chiralities: (a) armchair configuration, (b) zig-zag arrangement, and (c) chiral conformation [45]........................................ 14

6. (a) Molecular model of a; (b) HRTEM image of one end of a MWNT; (c) model of a

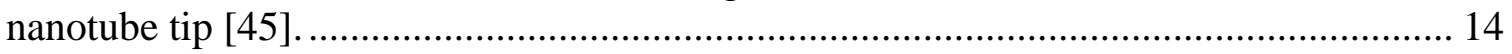

7. Left: Cup stacked, Middle: GG Tibbetts et al. [48], Right: Al-Saleh and Sundararaj ..... 15

8. CNF on the surface of a carbon fiber. Bamboo structure and hollow interior indicated. 16

9. MEMS-based measurement platform with mounted VGCNF [49]............................. 17

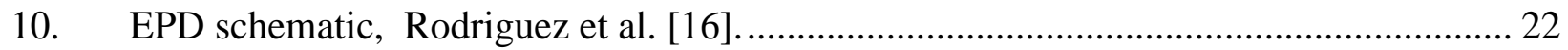

11. VARTM process used in the study by Song et al. [68]. ............................................. 23

12. SEM images of carbon fibers (CFs) with (a) SWCNTs and (b, c) MWCNTs deposited by

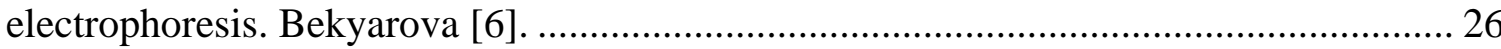

13. Single fiber EPD setup. Frame-mounted fibers being lowered into EPD tank (a); Fibers mounted on frame post deposition (b) ..................................................................... 31

14. Sized carbon fibers with O-CNFs deposited........................................................... 32 


\section{LIST OF FIGURES (continued)}

Figure

15. Single carbon fiber after one-stage $2 X$ concentration EPD. Layering shown in A; agglomerate indicated by white arrow in $\mathrm{B}$

16. Diagram of fiber fragmentation.

17. Two-parameter Weibull plot for sized, unsized, O-CNF deposited, and electric field fibers.

18. Break behavior for each fiber sample type.

19. Interfacial shear strength for each sample type. Error bars represent one standard deviation.

20. Percent difference compared to base sized fibers.

21. Sized fibers (a), unsized fibers (b), sized fiber exposed to 1 min electric field (c), unsized fibers exposed to 1 min electric field (d), sized fiber with 1 min O-CNF deposition (e), and unsized fiber with 1 min O-CNF deposition (f) . .............................................. 46

22. Sized fibers with 5 min O-CNF deposition. Irregular surface with high level of deposition (top), intermediate level of deposition (middle), and low level of deposition (bottom).

23. Exposed carbon fiber surface due to agglomeration of O-CNFs and deterioration of the epoxy sizing.

24. Fiber breaks for sized fiber (a), unsized fiber (b), sized fiber with 5 min O-CNF deposition (c), unsized fiber with 1 min O-CNF deposition (d), sized fiber exposed to 1 min electric field (e), sized fiber with 1 min O-CNF deposition (f), unsized fiber exposed to 1 min electric field (g).

25. Single carbon fibers representing $1 \mathrm{X}$ concentration (A), $2 \mathrm{X}$ concentration (B), and $2 \mathrm{X}-\mathrm{SS}$ (C) one-stage anode EPD approaches. In (B), arrows indicate agglomerates, while in (C) they indicate areas of increased surface disorder. 


\section{LIST OF FIGURES (continued)}

Figure

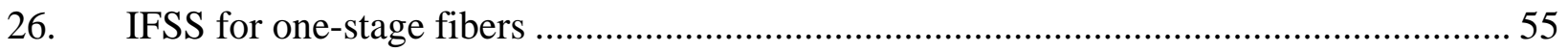

27. Two-stage A-CNF and one-stage O-CNF comparison ............................................. 58

28. Polarized light birefringes for two-stage A-CNF deposition fibers (A) and fibers acting as

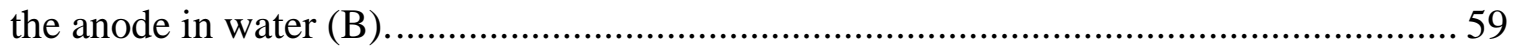

29. Break response under increasing applied tensile stress .......................................... 60

30. Frequency of tensile failure strength for base sized fibers, sized fibers acting as the anode, and sized fibers acting as the cathode. Field applied in water without presence of OCNFs or A-CNFs. Cathode data from Schaefer et al.[82]........................................... 61

31. Comparison of tensile failure samples for samples undergoing O-CNF EPD (top), 2S-2XSS EPD (middle), and anode exposure in water (bottom).

32. Magnified view of the anode fiber fragmentation failure surface. 63 


\section{LIST OF ABBREVIATIONS / NOMENTCATURE}

$\begin{array}{ll}\text { A-CNFs } & \text { Amine-Functionalized Carbon Nanofibers } \\ \text { CNTs } & \text { Carbon Nanotubes } \\ \text { CVD } & \text { Chemical Vapor Deposition } \\ \text { EPD } & \text { Electrophoretic Deposition } \\ \text { FESEM } & \text { Field Emission Scanning Electron Microscope } \\ \text { FT-IR } & \text { Fourier Transform Infrared Spectroscopy } \\ \text { IFSS } & \text { Interfacial Shear Strength } \\ \text { MEMS } & \text { Microelectromechanical Systems } \\ \text { MWCNTs } & \text { Multi-Wall Carbon Nanotubes } \\ \text { O-CNFs } & \text { Carboxylic Acid-Functionalized Carbon Nanofibers } \\ \text { SWCNTs } & \text { Single-Wall Carbon Nanotubes } \\ \text { TGA } & \text { Thermogravimetric Analyzer } \\ \text { TEM } & \text { Transmission Electron Microscope } \\ \text { VGCNFs } & \text { Vapor Grown Carbon Nanofibers }\end{array}$




\section{CHAPTER 1 \\ INTRODUCTION}

\subsection{Motivation and Scope}

The mechanical properties of carbon fabric-reinforced composites depend on those of the individual constituents and the degree to which they interact. This interaction occurs at the interface of the matrix and reinforcing fibers. Accordingly, the interface plays a pivotal role in stress transfer, the degree of which determines important mechanical properties including strength, stiffness, and weight. These considerations are vital in the design of composite components, which makes interface enhancement a critical research interest.

To study the interface, researchers have developed three well-known methods: microbond, pull-out, and fragmentation. These approaches, reviewed in detail by HerreraFranco and Drzal [1], characterize interface quality by determining the average interfacial shear strength (IFSS) of single fibers embedded in matrix. Often, they are employed to assess how altering the interface affects IFSS values. For example, increases in IFSS ranging from $17 \%$ to 217\% using the single fiber pull-out technique were reported for carbon fibers treated with acidic or basic surface oxidations by Ramanathan et al. [2].

Previously, Hughes [3] reviewed the carbon fiber-epoxy interface and discussed the factors impacting interface performance. The findings suggested that interfacial interaction could be enhanced by increasing fiber surface disorder and decreasing fiber diameter in order to increase the interaction surface area. Remarkably, Hughes provided an insightful strategy for designing future advanced composites. These concepts are actively being realized with the advent of nanotechnology in manufacturing composite materials; currently, researchers are

developing novel approaches such as chemical vapor deposition (CVD) and electrophoretic 
deposition (EPD) to place nanoparticles at the interface in order to increase surface roughness and surface area [4-8].

Due to geometry and superior mechanical, electrical, and thermal properties, carbon nanotubes (CNTs) have been incorporated at the interface to increase fiber-matrix interaction. Thostenson et al. [9] used CVD to grow CNTs on the carbon fiber surface, and predicted that local stiffening of the matrix near the interface enhanced load transfer. Zhang et al. [10] used the microbond approach to test CNT-coated carbon fibers in epoxy matrix. Reporting a $150 \%$ increase in IFSS, these authors attributed the enhancement to potential chemical bonding, increased fiber surface area, and mechanical interlocking. Also, Qian et al. [11] tested similar materials using the pull-out method and reported a 60\% IFSS increase. The fiber fragmentation method is particularly helpful when comparing the effect of surface modification on the stress transfer between ductile matrices and brittle fibers [12]. Several researchers have used this approach to compare the effect of adding CNTs to the interface $[5,9,13]$. Notably, Qian et al. [5] reported a 150\% increase in IFSS for silica fibers covered with CVD grown CNTs in poly(methyl methacrylate) (PMMA). Although leading to increases in the IFSS, CVD typically limits part geometry and damages the fiber surface which in turn decreases tensile strength. These limitations may be overcome through the use of special processing conditions [4], but currently, CVD is challenging for industrial application due to its expense and scalability concerns.

As a currently less-expensive and simpler to process material than CNTs, carbon nanofibers (CNFs) provide an economic opportunity for reinforcing the fiber-matrix interface. Palmeri et al. [14] used shear mixing to incorporate CNFs into the matrix of quasi-isotropic and unidirectional carbon fiber-reinforced composites to obtain increases in their short beam shear 
strengths. Recently, researchers have used EPD to place nanoparticles on conductive and nonconductive substrates [15-18]. In this method, particles are dispersed in a liquid (typically water) medium and an electric field is applied. Due to surface charge, the particles are deposited on a counter electrode, and Boccaccini et al. [15] and Biest et al. [19] reported that deposition is affected by electrode distance, electric field potential, and field duration. Rodriguez et al. [16] employed EPD to deposit functionalized CNFs onto the surface of carbon fabric layers. These hybrid CNF/carbon fiber layers were then used to manufacture multiscale composites with improved laminate properties such as interlaminar shear strength (ILSS) and electrical conductivity [7].

While researchers have successfully used both CVD and EPD approaches in the laboratory setting, it is the potential flexibility and scalability of the EPD process in water that makes it an intriguing area of research. Notably, Zhang et al. [17] used EPD to deposit multiwall carbon nanotubes (MWCNTs) on the surface of glass fibers and employed the fragmentation method to determine the average IFSS. While enhancing the IFSS by $30 \%$, the deposited MWCNTs also improved the electrical conductivity of the fibers. Park et al. [18] used EPD to deposit exfoliated graphene nanoplatelets onto the surface of carbon fibers and determined the IFSS over a range of applied voltages. They reported that the IFSS decreased for applied fields up to $30 \mathrm{~V}$. The investigators also noted the potential for slip planes to form at the interface; thus weakening the fiber-matrix interaction even though increases in substrate surface roughness and surface area had been achieved. Furthermore, Lee et al. [8] used a one-stage EPD approach to compare the ILSS of composites made from carbon fiber layers acting as either the anode or cathode during nanoparticle deposition. Interestingly, an ILSS increase of 13\% was reported for MWCNT-reinforced samples with the fibers acting as the cathode, while ILSS decreases were 
measured for anode deposition based samples. Alternatively, Rodriguez et al. [7] reported a 10\% ILSS increase for composite laminates manufactured from carbon fabric undergoing a two-stage EPD process using amine-functionalized carbon nanofibers (A-CNFs). Because of these results, it is clear that EPD is an effective method for placing nanoparticles at the interface; however, the methodology to effectively reinforce the interface requires further development. To determine how electrophoretically deposited CNFs affect the interface, it is imperative to investigate stress transfer at the simplest level—-the single fiber interface.

\subsection{Research Objectives}

To investigate the effects of electrophoretically deposited CNFs on the carbon fiberepoxy matrix interface, single fiber interface testing was performed. The aim of this research work was to obtain a fundamental understanding of the effects of adding functionalized CNF using EPD to a single carbon fiber-epoxy matrix interface. These findings seek to illustrate how using EPD to deposit functionalized CNFs affects interface shear strength, fiber tensile strength, and interface morphology. With these results, it is intended that the methodologies for using EPD to place nanoparticles at the interface may be improved in order to produce composites with superior stiffness, strength, and decreased weight.

In the current work, EPD was used to deposit carboxylic acid-functionalized CNFs (OCNFs) and A-CNFs on the surface of single carbon fibers. Using the fiber fragmentation technique and the shear-lag analysis of Kelly and Tyson [20], the stress transfer at the single fiber interface was characterized by determining the IFSS for different fiber surface treatments. For the O-CNF investigation, samples for sized, unsized, O-CNF deposited sized, and O-CNF deposited unsized carbon fibers were tested. A-CNF investigation was completed for sized carbon fibers acting as the anode during EPD for single (1X), and double (2X) concentrations of 
nanoparticles in water. Additionally, results for fibers acting as both the anode and cathode during a two-stage A-CNF deposition process are provided. Finally, the effects of EPD were investigated by testing fibers acting as the cathode or anode in water without the presence of OCNFs or A-CNFs. Weibull analysis of single-fiber tensile failure was performed to account for scale effects along the fiber length and support IFSS estimation [21, 22].

This investigation is presented as follows. Chapter 2 begins by providing a brief review of interface considerations and testing methods, and continues with an overview addressing the discovery of CNTs along with a presentation of nanoparticle morphology and general properties. Current methods for incorporating these nanoparticles as reinforcement in composite materials along with common limitations are presented to provide the appropriate scope for considering the simplified single fiber interface. A brief review of the process to functionalize CNFs is presented in Chapter 3, along with the experimental methods and techniques for depositing the functionalized CNFs on the surface of single carbon fibers and determining the average IFSS. The results for O-CNF deposited and A-CNF deposited fibers are provided in Chapter 4, and the interface is characterized using an optical microscope and field emission scanning electron microscopy (FESEM). The investigation is concluded in Chapter 5 with a presentation of additional research considerations. 


\section{CHAPTER 2}

\section{LITERATURE REVIEW}

\subsection{Interface Characterization}

Investigating the carbon fiber-matrix interface is a critical step in characterizing stress transfer in advanced composite materials. By linking constituent properties, the interface serves as an important determinant for improving overall composite strength, stiffness, and weighteach of which is an influential design parameter. Thus, it is imperative that the interface be tailored to enhance constituent interaction.

Noted by Hoecker and Karger-Kocsis [23], the inherent three-dimensional stresses that arise within laminate scale samples are quite complicated; thus, single fiber testing has been adopted to simplify analysis. Currently, the most common approaches for analyzing the single fiber-matrix interface are the pull-out, microbond, and fragmentation methods.

\subsubsection{Single Fiber Pull-Out}

The single fiber pull-out technique for determining IFSS involves embedding the end of a fiber in a small segment of matrix that is typically block or disk shaped and appropriately thin enough to allow for the fiber to be debonded. Testing is completed by holding the matrix section in place and gradually loading the fiber axially in tension in order to pull it from the matrix segment. The load and displacement are recorded until the fiber either pulls free of the matrix segment or fractures, which often occurs when the matrix segment is too thick or the fiber is weak. The interface strength is evaluated by relating the tensile stress on the fiber, $\sigma_{f}$, with the shear stress present at the fiber-matrix interface. The shear strength, $\tau_{I F S S}$, is determined by assuming uniform stress distribution along the embedded fiber length and determining the fiber diameter, $d$ : 


$$
\tau_{I F S S}=\left(\frac{\sigma_{f}}{2}\right)\left(\frac{d}{L}\right)
$$

where $L$ is the fiber embedded length. This test is illustrated in Figure 1.

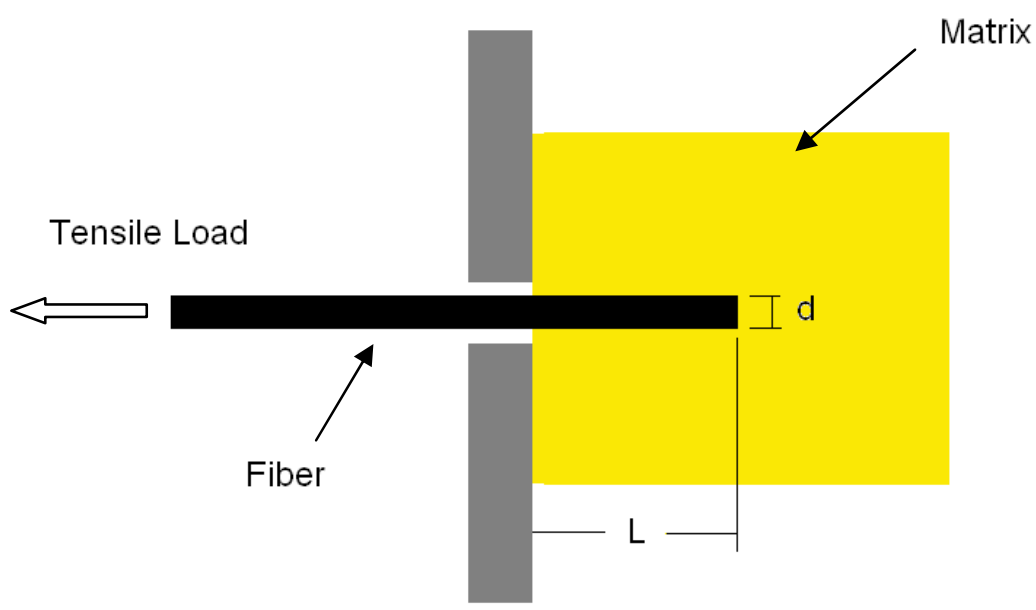

Figure 1: Schematic of fiber pull-out method

As noted by Herrera-Franco and Drzal [1], the pull-out approach allows for the use of many different fiber-matrix combinations and recording of the force at the moment of debonding. The pull-out samples, however, are difficult to manufacture due to the embedded length of the fiber being limited by fiber strength. In advanced composites-where fiber diameters range from 5 to $50 \mu \mathrm{m}$ — the embedded length may be limited to as little as 50 to 100 $\mu \mathrm{m}$ to avoid fiber fracture.

\subsubsection{Microbond}

Another approach to determining the average IFSS in single fiber composites is the microbond method, wherein a single fiber is sheared from a droplet of cured matrix. The samples are typically prepared by dipping a small diameter wire in matrix and then lightly 
touching the single fiber in order to deposit micron-sized droplets. The average IFSS is determined similarly to the pull-out method. The microbond method is depicted in Figure 2.

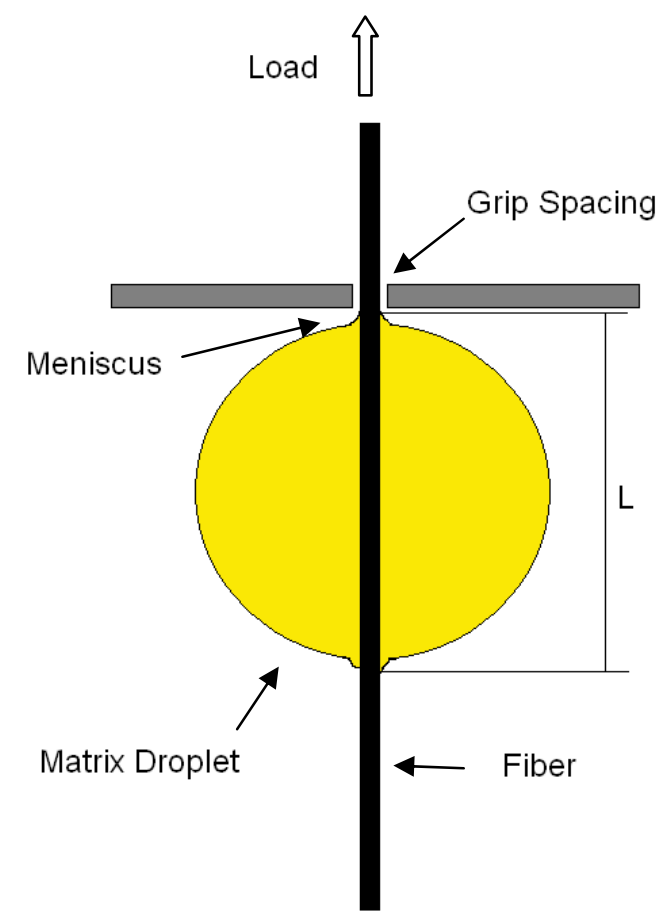

Figure 2: Microbond method.

This technique is advantageous because it may also be applied to many fiber-matrix combinations. However, the use of the microbond method is limited by several considerations. As with the pul-lout method, the microbond method requires careful control of the fiber embedded length to prevent fiber tensile failure before debonding occurs. The shape of the resin droplet may also affect the stress condition along the embedded length, and the presence of a meniscus (shown in Figure 2) occurring as the result of fiber surface wettability may further complicate measuring the embedded length accurately [24-26]. The meniscus may also induce early debonding which would undervalue the average IFSS. Due to fiber diameters becoming smaller in advanced composite manufacturing, the droplets also become difficult to create and test at the required diameters. The mechanical properties of the droplet may also be impacted by 
the volatility of the curing agent within the resin-hardener system. Rao et al. [27] proposed that the interfacial properties of the matrix may be impacted by variations in curing agent density. Another significant consideration is the grip spacing. The grip distance determines the focus of stress within the droplet, and large variations in grip spacing appear to affect the recorded IFSS $[1,28]$. Due to these limitations, the data obtained for a given fiber-matrix combination is typically scattered and contains large standard deviations. While the approach remains experimentally difficult, enhanced stress analysis along the fiber embedded length has been performed by Nishikawa et al. [29], who developed a simulation for evaluating microbond test results.

\subsubsection{Single Fiber Fragmentation}

The fiber fragmentation test was first employed by Kelly and Tyson in 1965 [20] to evaluate the properties of tungsten fibers embedded in a copper matrix. In this method, a brittle fiber is placed in a ductile matrix and tensile force is applied to induce shear stresses at the fibermatrix interface. These shear stresses cause tensile stress within the fiber. During the loading process, the embedded fiber will break continuously as stress is transferred from the matrix. After sufficient loading has been applied, the embedded fiber will stop producing new breaks, since the individual fragment lengths have become too small for the matrix to impart the stress required to cause fracture. This condition is referred to as "saturation" and represents the maximum stress transfer between matrix and fiber. The average IFSS is determined by assuming a constant state of shear stress along the short fiber segments and constant fiber diameter. The main limitations of this approach are that the matrix must have a sufficiently high strain limit to allow for fiber saturation to occur, and it must also maintain appropriate toughness to avoid sample failure due to fiber fracture. The matrix is also required to be transparent in order to see and measure the fiber fragments. While these limitations exist, the approach provides a large 
body of information for statistical purposes, presents an in situ failure process, and is generally practical at the experimental level. Additional investigation has been performed by Paipetis et al. [30] using Raman experiments to map stress along the embedded fiber lengths under increasing tensile stress. This approach, along with numerical simulation [31], indicates the potential for obtaining meaningful interface results from this method. A detailed presentation of the single fiber fragmentation test is provided in Chapter 3: Technical Approach.

Each of these methods has been employed by researchers to investigate a variety of interfaces. Bogoeva-Gaceva et al. [32] used the pull-out method to determine that sized carbon fibers may produce higher interfacial shear strength than unsized fibers, and Gaur et al. [33] used the microbond approach to investigate hydrothermal aging effects on bond strength for several fiber types in epoxy and polyethylene resin systems. Additionally, several researchers have used the fiber fragmentation test to investigate the effect of sizing, resin hardener, and environmental conditions on interface stress transfer [34-39]. Further review of these testing methods has been performed by Herrera-Franco and Drzal [1], Rao et al. [27], Hamada et al. [40] and Zhandarov and Mäder [12].

\subsection{Morphology and Properties of Carbon Nanotubes and Carbon Nanofibers}

\subsubsection{Background}

The discovery of single wall carbon nanotubes (SWCNTs) is largely attributed to the published works of Iijima [41, 42] and Bethune et al. [43] in Nature articles from the early 1990s. However, as noted by Monthioux and Kuznetsov [44] and in the review of Terrones [45], it has since been determined that the actual discovery could be shared with Oberlin et al. [46]. In an attempt to analyze and determine the growth mechanism of carbon fibers produced using high temperature pyrolosis of benzene and ferrocene, Oberlin et al. unknowingly produced the first images of single wall and multi-wall carbon nanotubes (MWCNTs) using high-resolution 
transmission electron microscopy in 1976. At the time, they noted the ability to produce fibers having diameters less than $100 \mathrm{~nm}$, but the implications of the accomplishment went largely unnoticed by a research community more focused on investigating the properties of micron sized fibers. Their original work is depicted in Figure 3. Then, in 1991, Iijima reported the observation of what appeared to be carbon forms comprised of rolled graphitic sheets that formed tube shaped cylinders with diameters ranging in the tens of nanometers. These concentric 'microtubles' contained an interlayer spacing of approximately 3.4 angstroms and came to be known as MWCNTs [41]. The official discovery of SWCNTs came shortly thereafter, as Iijima and Ichihashi [42] and Bethune et al. [43] independently reported the creation of carbon tubes comprised of a single carbon shell using an electric arc discharge technique and metal catalyst. With the presentation of these new nano-scale carbon forms and a novel production method came a surge of research interest; the number of CNT related publications expanded from less than 200 in 1994 to more than 1500 per year in 2001 [45]. The era of nanotechnology had begun. 


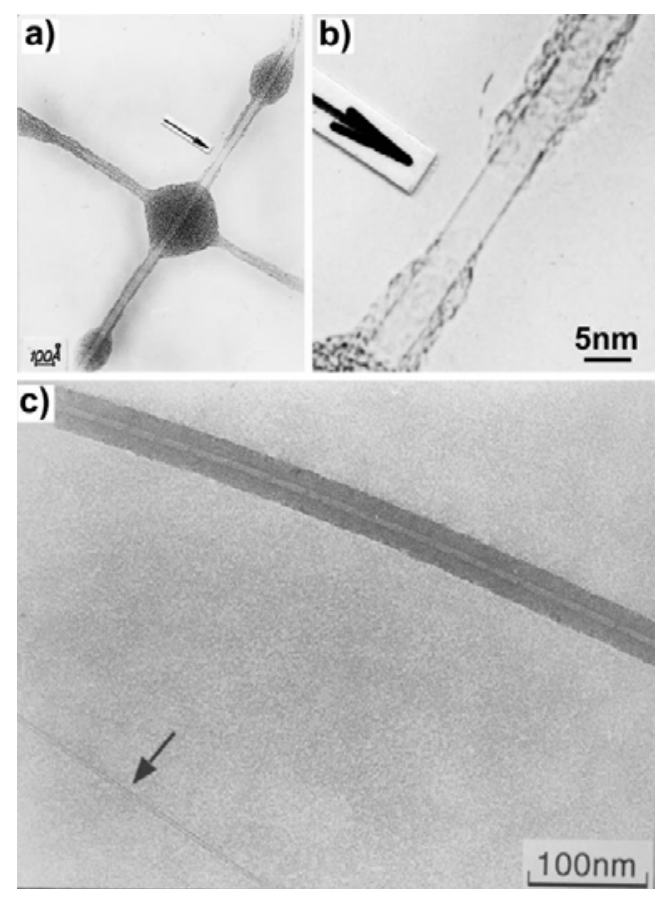

Figure 3: (a) HRTEM image of two crossing SWNTs coated with amorphous carbon; (b) Higher magnification of (a); (c) HRTEM image showing a MWNT upper region together with a SWNT indicated by an arrow [45].

\subsubsection{Carbon Nanotube Morphology}

The properties of CNTs are largely dependent on morphology and the degree of graphitization. CNTs can be visualized as a single layer of hexagonal graphene atoms that has been rolled to form a tube. This tube may be rolled to produce chiral and non-chiral configurations. For the non-chiral configurations, two carbon-carbon atoms (C-C) on opposite sides of a hexagon may lie perpendicular to the tube axis (armchair) or parallel to the tube axis (zig-zag), while chiral configurations are defined as those that display any other angle. Illustrations are provided in Figure 4 and Figure 5. As reviewed by Terrones [45], the chiral vector $-\mathrm{Cn}\left(\mathrm{Cn}=m \mathrm{a}_{1}+n \mathrm{a}_{2}\right)-$ may be used to determine the diameter of the tube:

$$
d=\frac{a \sqrt{m^{2}+m n+n^{2}}}{\pi}
$$


where $a$ is the graphite sheet lattice constant (1.42 $\mathrm{x} \sqrt{3} \AA$ ) and $m$ and $n$ are lattice coordinates. Further details regarding unitary vectors $\mathrm{a}_{1}$ and $\mathrm{a}_{2}$, tube diameter, and tube chirality are discussed in the review. The morphology of a SWCNT, MWCNT, and end-cap model for a MWCNT is shown in Figure 6.

Of important note is that armchair and some zigzag ( $m$, and $n$ being a multiple of 3) configurations are metallic in nature. SWCNTs typically have diameters between 0.6 and $2 \mathrm{~nm}$ while MWCNT diameter ranges from approximately 5 to $50 \mathrm{~nm}$ [47]. At present, the most common methods for synthesizing CNTs are electric arc discharge, laser vaporization, and pyrolosis of hydrocarbons over metals.

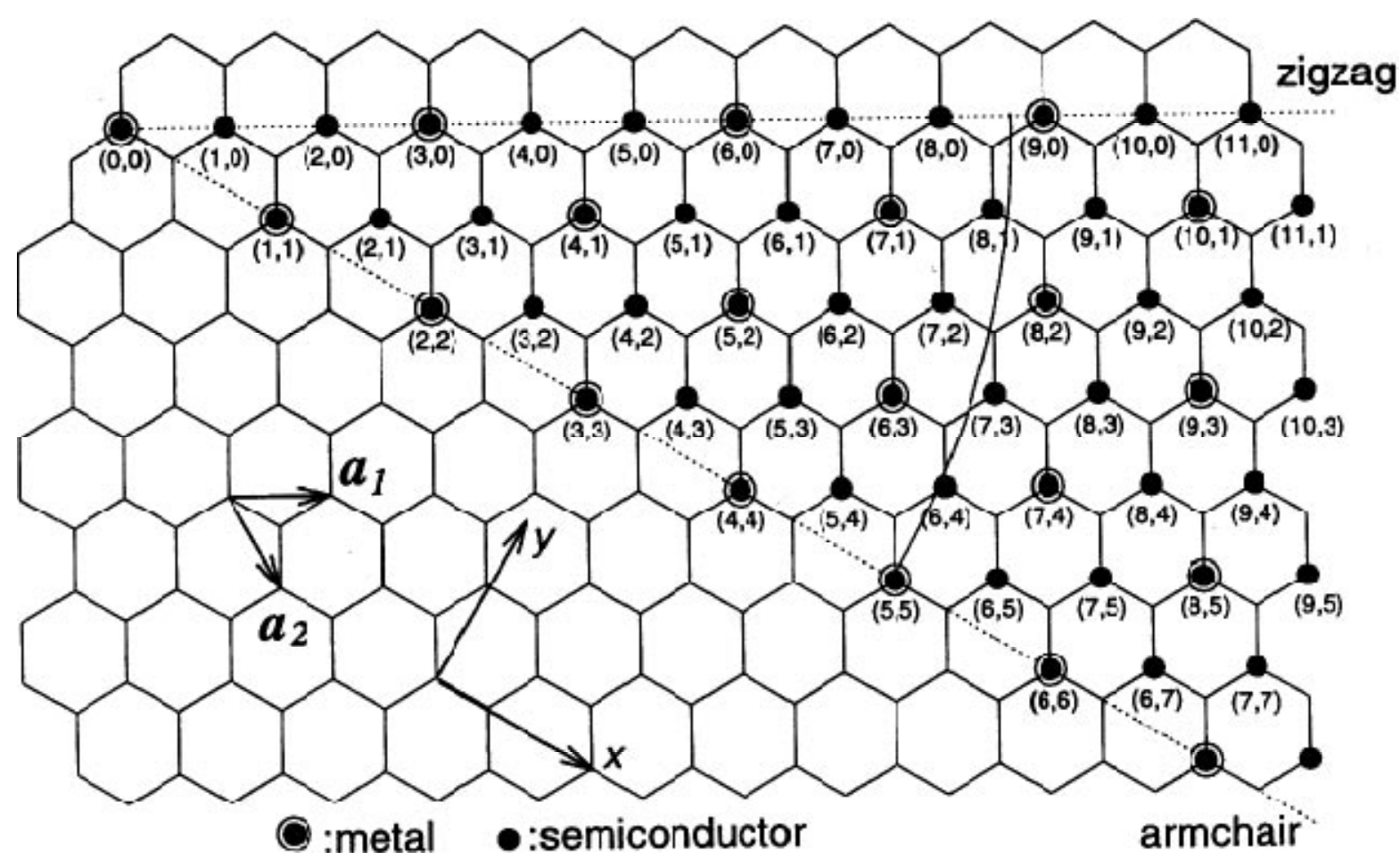

Figure 4: Graphene sheet [45]. 

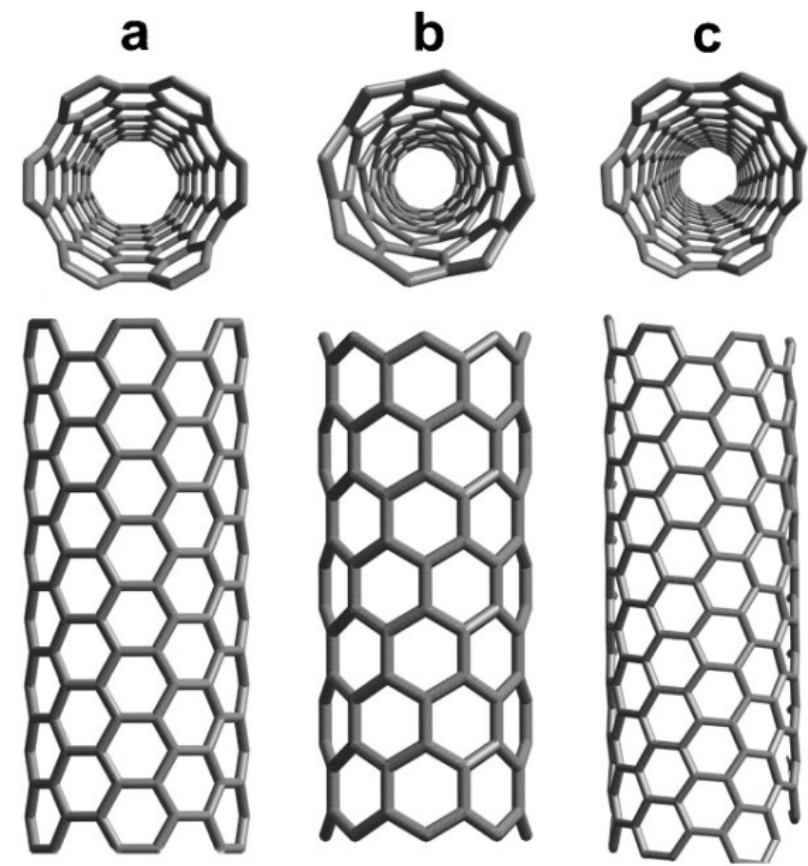

zigzag

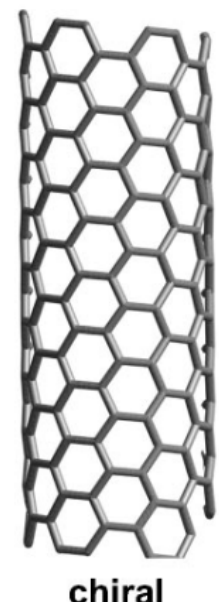

Figure 5: Molecular models of SWNTs exhibiting different chiralities: (a) armchair configuration, (b) zig-zag arrangement, and (c) chiral conformation [45].

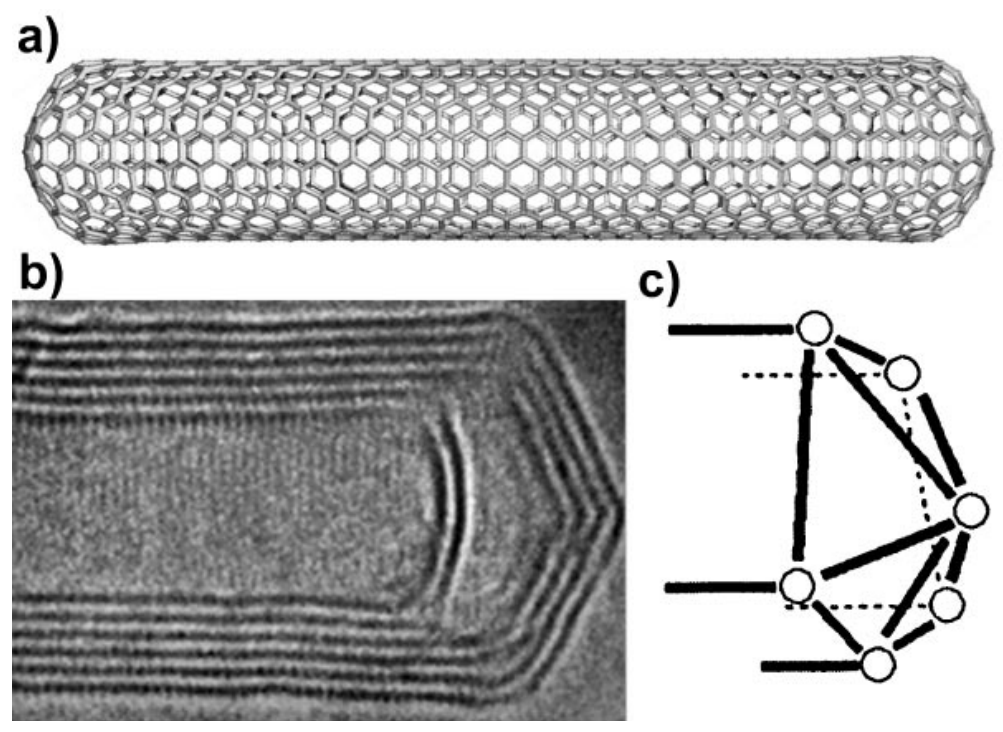

Figure 6: (a) Molecular model of a; (b) HRTEM image of one end of a MWNT; (c) model of a nanotube tip [45]. 


\subsubsection{Carbon Nanofiber Morphology, Production, and Properties}

Carbon nanofibers are nano-scale fibers (diameter in nanometers, length in microns) that typically exhibit aspect ratios ranging from 250 to 2000 . As indicated in Table 1 , this is roughly between the values for CNTs and carbon fibers; accordingly, other CNF properties exhibit similar behavior. A common method for producing CNFs is to perform catalytic chemical vapor deposition of a hydrocarbon or carbon monoxide above a metal surface or metal alloy catalyst in a reactor at a temperature between $500{ }^{\circ} \mathrm{C}$ and $1500{ }^{\circ} \mathrm{C}$. This process produces vapor grown carbon nanofibers (VGCNFs) [47]. Altering the feedstock and catalysts will change the morphology of the VGCNFs created, and it is possible to produce linear, twisted, and helical conformations. Of note is that CNFs have a hollow core caused by the stacking of graphite planes to create different morphologies such as cup stacked, bamboo-like, and parallel structure. An illustration of the CNF interior nature is provided in Figure 7, and an image of bamboo-like CNF morphology is shown in Figure 8 . Also visible is the hollow CNF interior.
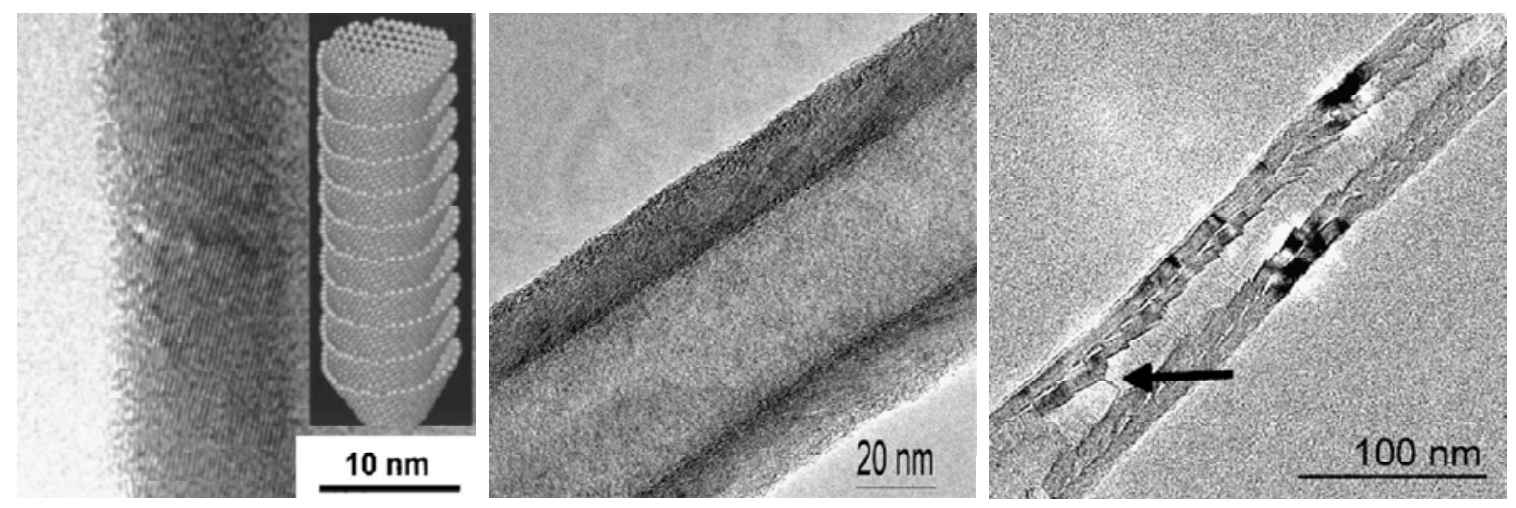

Figure 7: Left: Cup stacked, Middle: GG Tibbetts et al. [48], Right: Al-Saleh and Sundararaj [47]. 


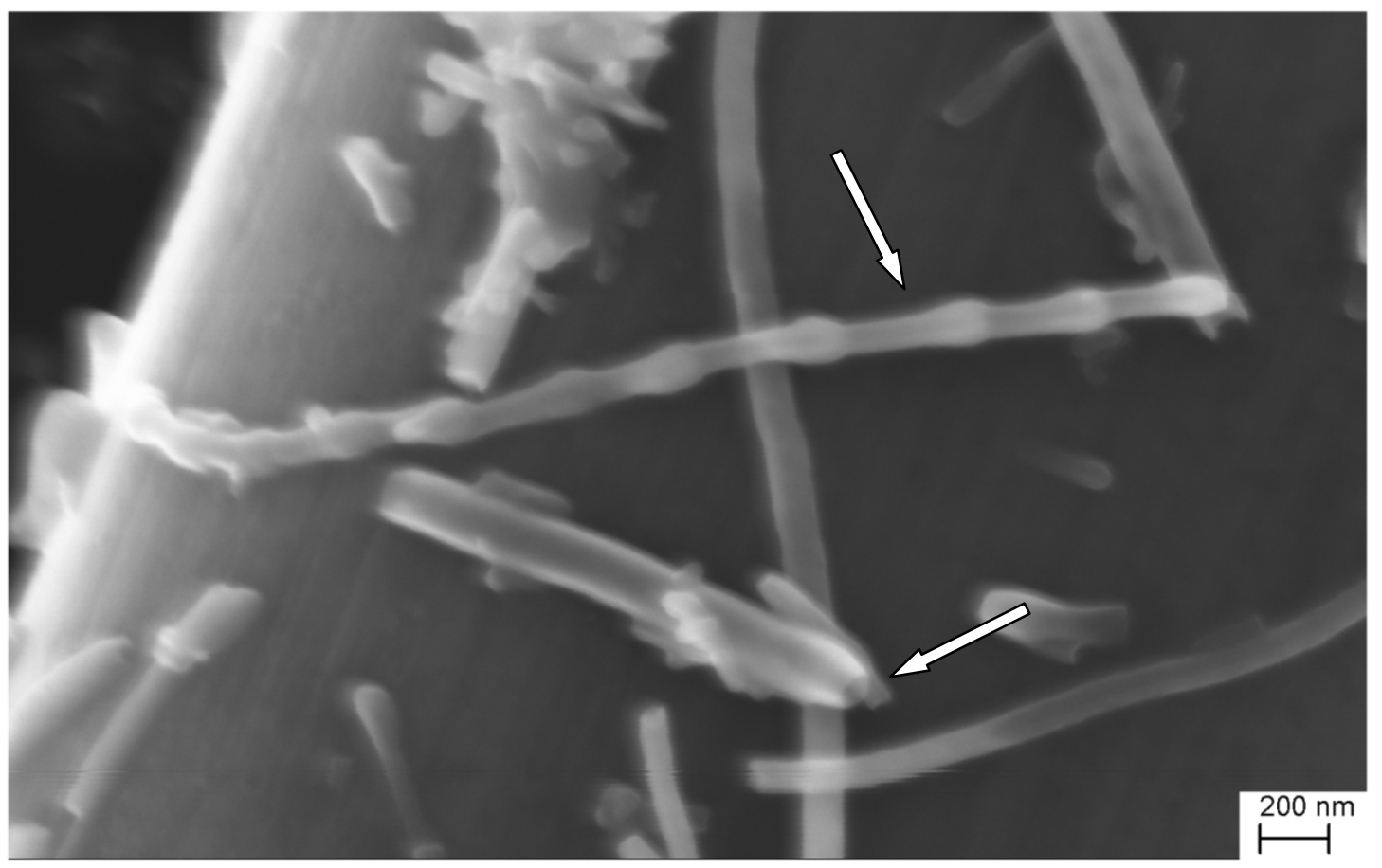

Figure 8: CNF on the surface of a carbon fiber. Bamboo structure and hollow interior indicated.

Because of these different morphologies and variations in the interior CNF structure, measuring the mechanical properties of CNFs has proven to be a challenge for researchers. Ozkan et al. [49] recently used microelectromechanical systems (MEMS) tools to directly measure the tensile strength and modulus of single CNFs; they used scanning electron microscopy (SEM) and transmission electron microscopy (TEM) imaging to characterize the fracture morphology. It was noted that the fiber strength decreased with increasing graphitization temperature, which is potentially due to reduction of the exterior turbostratic layer and an increase in the order of the graphene planes. Further, the elastic modulus increased from 180 to 245 GPa. The testing setup is depicted in Figure 9. 


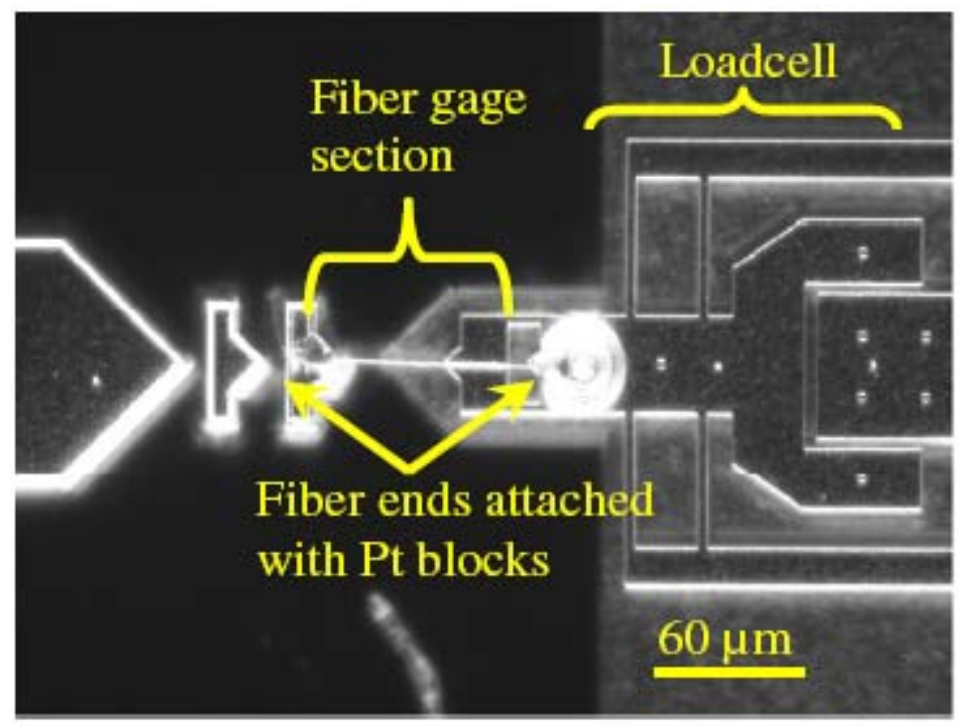

Figure 9: MEMS-based measurement platform with mounted VGCNF [49].

The increase in modulus and decrease in strength due to processing temperature indicates that the properties of CNFs may be altered through additional treatments. To date, researchers have used oxidative treatments [50], etching [51], and amine functionalization [52, 53] to improve CNF compatibility with the matrix, and others have used heat treatments to achieve increased mechanical and electrical properties [54]. Of note, Finegan et al. [51] determined that adhesion between the fiber and matrix was improved by using air or $\mathrm{CO}_{2}$ to etch the $\mathrm{CNF}$ surface to create increased CNF surface area and roughness. While the mechanical properties of the overall composite were increased, it was noted that heat treated CNFs typically exhibit poor interaction with the matrix due to increased graphitization of the surface layers which decreased chemical reactivity. Tibbetts et al. [48] reviewed the effect of temperature on CNF graphitization to determine how increasing temperature affects graphite crystallinity. It was shown that treatments at $1500{ }^{\circ} \mathrm{C}$ created graphitized layers on the CNF exterior that aligned with the fiber axis, while treating $\mathrm{CNF}$ at $2800{ }^{\circ} \mathrm{C}$ caused the nested graphite planes to recrystallize which limited the physical properties. Increased optimization of the heat treatment 
approaches determined that the best properties were achieved for CNF with fully graphitized layers along the fiber axis [54]. In order to enhance CNF reactivity with the matrix, aminefunctionalization has been used to place amine groups on the CNF surface, and as shown in the work of Prolongo et al. [53], amine-functionalized CNFs may be used to enhance composite thermal and mechanical properties. Additionally, Soo-Na et al. [52] reported the potential for amine-functionalization to enhance the dispersion and interfacial adhesion in epoxy resin.

\subsubsection{Properties of Carbon Nanotubes and Carbon Nanofibers}

A comparison between the geometry and mechanical properties of VGCNFs, SWCNTs, and MWCNTs compared to a standard carbon fiber was done by Al-Saleh and Sundararaj [47] and is presented in Table 1. A cost comparison at the time of writing for each type of conductive nanoparticle is provided in Table 2.

Table 1: Properties of VCGNFs, SWCNTs, MWCNTs, Al-Saleh and Sundararaj [47].

\begin{tabular}{lcccc}
\hline \multicolumn{1}{c}{ Property } & CNF & SWCNT & MWCNT & CF \\
\hline Diameter (nm) & $50-200$ & $0.6-1.8$ & $5-50$ & 7300 \\
Aspect ratio & $250-2000$ & $100-10000$ & $10-10000$ & 440 \\
Tensile strength (GPa) & 2.92 & $50-500$ & $10-60$ & 3.8 \\
Tensile modulus (GPa) & 240 & 1500 & 1000 & 227 \\
\hline
\end{tabular}

Table 2: CNT/Graphene Pricing [55] and CNF Pricing [56].

\begin{tabular}{ccc} 
SWCNTs (90\% pure) & MWCNTs (95\% pure) & CNFs \\
\hline$\$ 95-\$ 200 /$ gram & $\$ 5-\$ 15 /$ gram & $\$ 0.46 /$ gram
\end{tabular}

Compared to a standard carbon fiber, nanoparticles exhibit significantly increased properties. This is partially due to a decreased presence of defects at the nano level as well as a greatly increased aspect ratio. The reduced physical dimensions of CNTs and CNFs greatly increase the surface area available for interaction within a composite structure, making them ideal for enhancing strength and stiffness while minimizing weight. Aside from individual CNT 
and CNF properties, CNFs are currently much cheaper to produce than CNTs; it is the combination of enhanced properties, economic production, and ease of use that makes CNFs intriguing. Until CNTs can be produced at affordable rates, CNFs offer a considerably more economic option for laboratory and industry use. It is important to note that all nanoparticle production methods produce a bulk volume that contains varying levels of purity and imperfections [57].

\subsection{Applications in Advanced Composites}

\subsubsection{Processing}

At the bulk level, researchers have investigated using CNTs and CNFs as reinforcement in composite materials. In the axial direction, fiber reinforced composite properties are governed by fiber properties. The transverse direction, however, is greatly controlled by matrix dominated properties. Nanoparticles provide an attractive opportunity for enhancing these out-of-plane properties.

In order to take advantage of the unique individual nanoparticle properties, the particles must first be dispersed into a stable medium. Dispersion is often challenging, as the small particles tend to agglomerate due to intermolecular Van der Waals forces. These agglomerates will limit overall composite performance [58]. Nanoparticles can be dispersed in a matrix, in solution, or grown/deposited on a fabric surface depending on the composite manufacturing requirements.

Three methods are currently used to obtain a stable dispersion of CNTs and CNFs in a matrix. They are solution blending, melt blending, and in situ polymerization. Solution blending is most often used when the volume of materials is small. Initially, CNTs are dispersed in a solvent using sonication, which may take considerable time. Moniruzzaman and Winey [57] noted in their review of CNT composite processing that exposure to extended sonication time 
reduces CNT length and that this could greatly affect the influence of the particles in the composite. Once dispersed, the nanoparticles are collected and subsequently mixed with a polymer and the mixture can be poured into a mold or used as the matrix in a resin-addition process for fabric based composites. Often, surfactants are used to aid with dispersing high loadings of particles. An undesirable consequence, however, is that the surfactant remains in the final composite and must be removed. It has been shown that for similar loadings of CNTs, composites containing surfactant-assisted dispersion exhibited lower thermal conductivities [57]. However, Gong et al. [59] reported increases in glass transition temperature and elastic modulus in CNT polymer composites that were attributed to the enhanced dispersion associated with using a nonionic surfactant.

Melt blending involves using elevated temperature and high shear forces to disperse the particles in a polymer matrix [60,61]. Compared to solution blending, melt blending typically achieves lower levels of dispersion and is limited to use with lower concentrations of CNTs and CNFs. At high loadings, the viscosity of the resin-particle mixture becomes too high for proper resin flow.

In situ polymerization is a commonly used method for dispersing CNTs and CNFs in epoxy composites. First, the nanoparticles are dispersed in a monomer. A hardener (curing agent) is then added to the monomer-nanoparticle blend and the mixture is added to a mold or incorporated into a fabric preform and subsequently cured. This method allows for potential covalent bonding to occur between the nanoparticles and the polymer matrix. Functionalizing the surface of the nanoparticles increases the likelihood of potential bonding [57, 62].

Pristine CNTs are known to be insoluble in water. Accordingly, various surface modifications and functionalizations have been developed to address this issue. Zhu et al. [62] 
improved the dispersion of SWCNTs in an epoxy composite using acid treatments and fluorination at $1 \mathrm{wt} \%$ CNT loading, while Gojny et al. [63] used organic acids to oxidize the surface of MWCNTs to achieve reduced agglomeration. Dispersion of nanoparticles is a continuingly important research area.

Another approach for incorporating CNTs into composites is to use chemical vapor deposition to grow CNTs on the fiber surface. Garcia et al. [64] used chemical vapor deposition to grow CNTs on the surface of plain weave alumina fabric, and Qian [11] et al. grew CNTs on the surface of single carbon fibers using iron as the catalyst. In both cases, the fiber surface must first be coated with catalyst (typically metal) particles to provide the CNT growth locations. Second, the fibers are heated to above $750{ }^{\circ} \mathrm{C}$ in the presence of hydrogen and/or ethylene (for example), which promotes CNT growth. The flow rate of the gas additives can be altered to create CNTs of different lengths. The homogeneity of the surface grown CNTs is largely dependent on the size and dispersion uniformity of the precursor catalyst.

Of importance to this work, CNFs and properly prepared CNTs may be dispersed in a liquid medium for use in electrophoretic deposition. Researchers have used EPD to effectively manipulate nanoparticles and place them on conductive and non-conductive substrates [15-19, 65]. In this method, particles are dispersed in a liquid (typically water) medium and an electric field is applied. Based on surface charge, the particles will deposit on a counter electrode. Controlling the deposition time, solution concentration, and field potential determines the thickness of particle deposition $[15,19,66]$. If the nanoparticles are deposited on a fabric layer, the resulting multiscale fabric may be used to fabricate a composite laminate or panel. Rigueur et al. [67] presented a method for making thin MWCNT 'buckypaper' films using EPD that could be used to enhance composite laminates, while Rodriguez et al. [7] manufactured enhanced 
composites from fabric prepared by EPD of functionalized CNFs. Figure 10 is an illustration of a one-stage EPD process for depositing CNFs on a counter electrode, and further details regarding EPD are provided in Chapter 3: Technical Approach.

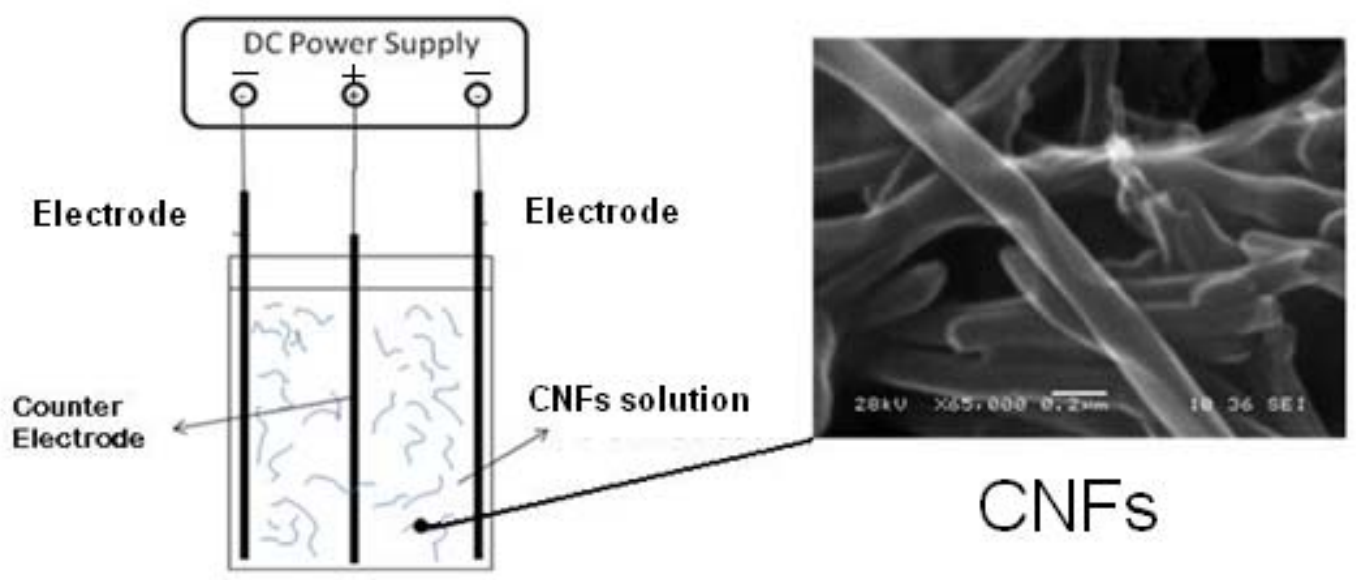

Figure 10: EPD schematic, Rodriguez et al. [16].

After dispersing the particles in a matrix/liquid medium, growing them on the fabric surface using CVD, or using EPD to place them on the substrate surface, a composite laminate can be made by pouring the nano-reinforced matrix into a mold and curing, by combining several nano-reinforced fabric layers and employing wet layup or a resin infusion process to create a panel, by adding the resin to individual fabric plies to make prepregs, or by a combination of these methods. Typically, vacuum assisted resin transfer molding (VARTM) or another resin infusion method is used in combination with single or double-sided tooling to infuse the nanoreinforced resin (or plain resin) into a woven fabric ply layup. A schematic for a typical VARTM layup is depicted in Figure 11. 


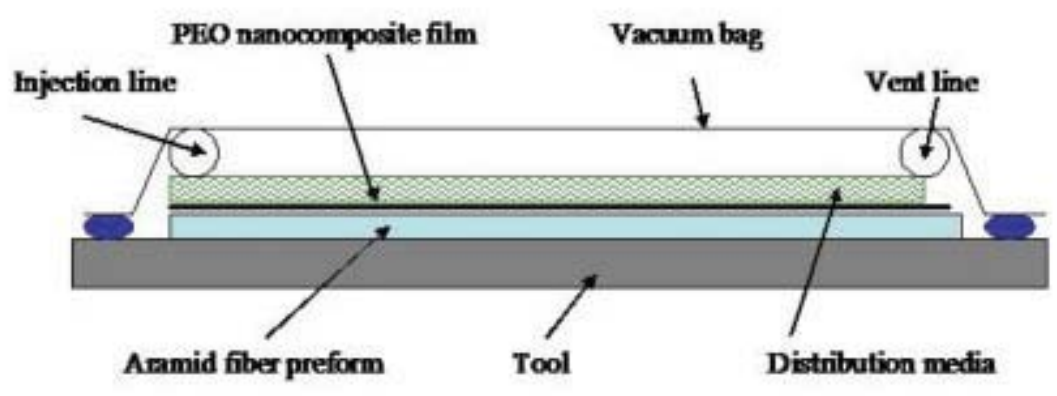

Figure 11: VARTM process used in the study by Song et al. [68].

\subsubsection{Properties of Nano-Reinforced Composites}

The discussion of nano-reinforced composite properties is defined by the material that is being reinforced. Thus, this review will focus on the two most commonly researched nanoreinforced materials: the polymer matrix and the reinforcing fabric.

\subsubsection{Polymer Nanocomposites}

A polymer nanocomposite is simply a resin-nanoparticle mixture that has been cured. Zhu et al. [62] reported a 30\% increase in tensile modulus and an $18 \%$ increase in tensile strength by effectively dispersing SWCNTs in an epoxy matrix and using acid treatments to functionalize the particles. Gong et al. [59] used surfactant-assisted processing to disperse CNTs in an epoxy matrix and reported an increase in elastic modulus of more than $30 \%$. This group also noted that the glass transition temperature rose by more than $20{ }^{\circ} \mathrm{C}$. Moisala et al. [69] investigated the electric percolation threshold of MWCNT and SWCNT reinforced epoxy composites. The percolation threshold is the point at which enough filler material is present to create an effective electrical network, which greatly increases the conductivity of the composite. After the percolation threshold is reached, addition of further filler material generally does little to increase conductivity. Percolation was achieved for both CNT types, with MWCNTs reaching 
percolation at $0.0025 \mathrm{wt} \%$ and SWCNTs at $0.05 \mathrm{wt} \%$. An increase in $10^{8} \mathrm{~S} / \mathrm{m}$ was reported for single and multiwall CNT-reinforced composites at percolation compared to the base epoxy specimen. Gou et al. [70] used SWCNT buckypaper and a hot press to produce composites with 20 to $30 \mathrm{wt} \%$ CNT loading. The storage modulus increased by 250 to $300 \%$. Interestingly, Yang et al. [71] recently combined MWCNTs and graphene nanoplatelets to produce composites with $35.4 \%$ higher tensile strength and a $147 \%$ increase in thermal conductivity.

A review of CNF composites by Tibbetts et al. [48] reported that CNFs addition to a polymer matrix can also greatly decrease the electrical resistivity. It was reported that Lafdi and Matzek [72] produced Epon 862 epoxy composites reinforced by surface oxidized VGCNFs with a modulus increase of approximately 3 times that of the base resin sample. Increases in compressive and tensile strength were also shown to be related to the wt\% of VGCNFs in the composite. Choi et al. [73] dispersed VGCNFs in epoxy to produce composites with increased storage modulus and glass transition temperature. Their work concluded that VGCNF reinforced epoxy composites appear to have a maximum tensile strength and Young's modulus at $5 \mathrm{wt} \%$ loading, and in general, composites with low viscosity had higher properties than those with elevated viscosity due to higher nanoparticle loading. Finally, Yang et al. [74] reported a ten order of magnitude increase in electrical conductivity for polystyrene reinforced by CNFs at 3 wt\%.

\subsubsection{Nano-Reinforced Fabric Polymer Composites}

Nano-reinforced fabric-polymer composites (also known as hybrid-layer composites, fiber-reinforced hierarchical composites, and multiscale reinforced-fabric composites) are a multiscale approach for enhancing material properties. By employing EPD or CVD to place nanoparticles on the fabric surface, researchers have created composite laminates with increased properties. Thostenson et al. [9] grew CNTs on the surface of carbon fibers using CVD and 
reported increases in IFSS using the single fiber fragmentation technique. It was noted that catalyst application and the CVD growth conditions caused a significant degradation of the fiber surface. Multiscale composites using CNTs were also created by Song [68], who used VARTM and aramid fabric layered with a polymer nanocomposite film, and increases in matrix dominated flexural and compressive properties as well as impact strength were reported. Bekyarova et al. [6] used EPD to deposit SWCNTs and MWCNTs on woven carbon fabric. This group noted a two-fold increase in out-of-plane electrical conductivity, while the in-plane conductivity remained unchanged after CNT addition. A 30\% increase in ILSS was also reported. Others have functionalized the surface of CNFs, employed EPD to deposit the particles on the carbon fabric layer surface, and manufactured multiscale composites with increased shear strength and electrical conductivity $[7,8,16]$. An image of CNTs deposited using EPD on carbon fibers is provided in Figure 12. 

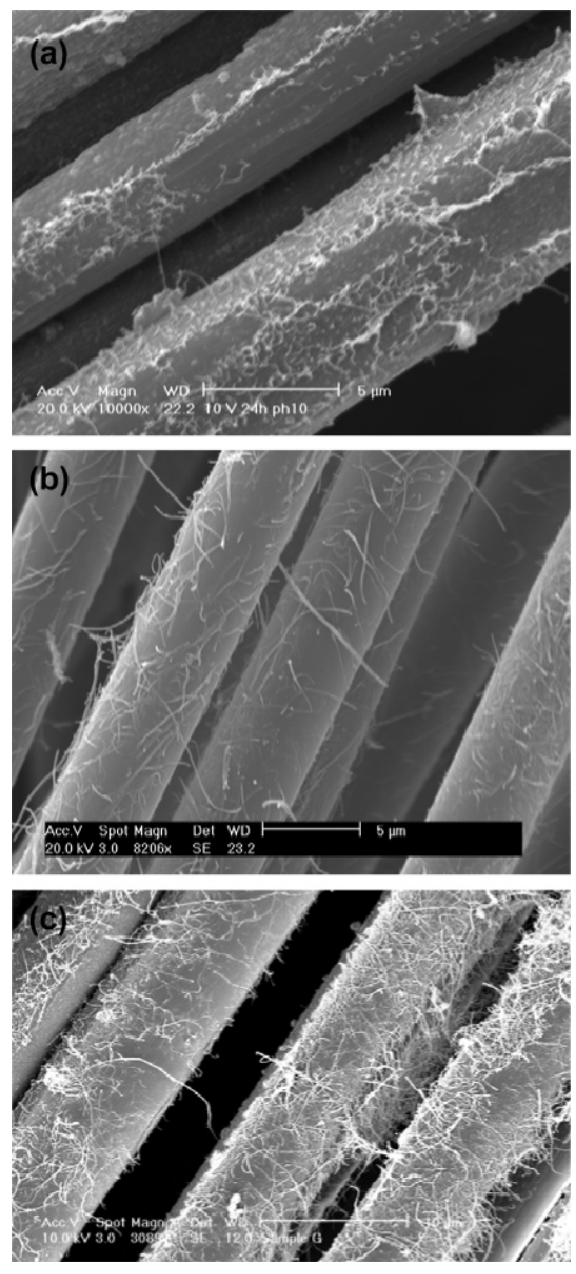

Figure 12: SEM images of carbon fibers (CFs) with (a) SWCNTs and (b, c) MWCNTs deposited by electrophoresis. Bekyarova [6].

\subsubsection{Limitations}

As indicated, researchers have used CNTs and CNFs to create composites with increased tensile modulus, shear strength, compression, and electrical conductivity. This makes these nanoparticles intriguing candidates for structural applications in the automobile, aeronautic, and aerospace fields where strength and stiffness to weight ratios govern the limiting criteria in design. While it appears that these nanoparticles may provide an effective means for enhancing composite properties, their use is currently limited by several factors. Common issues concerning the addition of nano-reinforced matrix to both molds and plain weave layups are the 
occurrence of nanoparticle agglomeration, nanoparticle filtration, and void formation. Agglomerations may form during cure, since it is typical for thermosetting resins to become less viscous before reaching the glass transition temperature which allows the fibers to move due to local viscous forces and van der Waals forces. Filtration of the nanoparticles may also occur if the resin is added to a fabric material. The nanoparticles may collect at the fibrous regions and agglomerate which prevents them from uniformly saturating the laminate, and this can create nanoparticle rich areas in the part [75]. Voids form due to volatiles in the resin, and often form in nanoparticle rich areas of the part as well. The presence of voids may indicate poor dispersion of the nanoparticles, loss of vacuum during cure, or inadequate resin volume or vacuum pressure. In general, these defects commonly form together, and all serve to decrease composite properties. The methods for placing nanoparticles on the fabric surface may also make scalability from the laboratory setting to an industry setting a challenge. CVD requires highly specialized processing conditions to prevent deterioration of the fiber tensile strength and limits the geometry of samples and parts [4]. It is also considerably more expensive than EPD in the lab setting.

Because of these factors, the analysis of nano-reinforced composite materials is difficult at the laminate scale. As indicated by the literature, many groups have performed similar work but obtained considerably different results. The discrepancies in manufacturing methods and conditions make understanding the reinforcing mechanisms of the nanoparticles a challenge. As indicated in Table 2, CNTs are considerably more expensive than CNFs, and these particles also require considerably more processing before they are usable (due to insolubility with water, impurities, and the bulk state at delivery). Before nanoparticles can be economically utilized in 
industry, a balance must be found between the cost of production, scalability, and potential property enhancement in composite materials.

In light of these considerations, it is important to understand the parameters affecting the use of nanoparticles within composite materials. By investigating the nature of stress transfer between the constituents, researchers may potentially enhance interaction through the use of nanoparticles at the fiber-matrix interface to create composite materials with improved mechanical, electrical, and thermal properties. Single fiber testing of nanoparticle-enhanced fiber-matrix interfaces may potentially provide the insight necessary for quickly developing novel methodologies to create nano-enhanced advanced composites. In order to practically connect research to application, it is important for investigations to be directed towards inexpensive and scalable approaches. As noted, an especially promising methodology is to use EPD to deposit functionalized CNFs on the carbon fiber surface. The remainder of this thesis focuses on presenting a fundamental comparison of the effects of electrophoretically deposited functionalized CNFs on the fiber-matrix interface. 


\section{CHAPTER 3}

\section{TECHNICAL APPROACH}

\subsection{Materials and Experimental Methods}

\subsubsection{Materials}

Pyrograf III PR 24 XT PS CNFs acquired from Applied Sciences, Inc. with a diameter range of $60-150 \mathrm{~nm}$, length range of $30-100 \mu \mathrm{m}$, and iron content less than 14,000 ppm (as provided by the vendor) were used. The CNFs were functionalized to introduce carboxylic acid groups or amine groups on the surface using the procedures outlined in the supporting material of Rodriguez et al. [16]. Characterization of the CNFs was performed using Fourier transform infrared (FT-IR) spectroscopy and a thermogravimetric analyzer (TGA). The details and confirmation of functional groups on O-CNFs and A-CNFs can be found in [76, 77].

Individual PAN-based carbon fibers having an average diameter of $5.2 \mu \mathrm{m}$, density of

$1.78 \mathrm{~g} / \mathrm{cm}^{3}$, average tensile strength of $5.48 \mathrm{GPa}$, and tensile modulus of $276 \mathrm{GPa}$ were pulled from IM7-6K tows and used for single fiber fragmentation and single fiber tensile tests. Fiber sizing was removed by placing fiber tows in a furnace at $500^{\circ} \mathrm{C}$ for $20 \mathrm{~min}$.

To manufacture the fragmentation samples, silicone molds were prepared by mixing KE 1300T mold making silicone and CAT 1300 hardener from ShinEtsu Silicones of America, Inc. in a 10:1 ratio by weight. The liquid silicone was poured into a master mold containing dog-bone shaped metal inserts and degassed at room temperature under full vacuum for approximately 30 min. The mold was then cured at $25{ }^{\circ} \mathrm{C}$ for $24 \mathrm{~h}$.

\subsubsection{Functionalization of CNFs}

Two procedures were used to create CNFs with increased surface functionality. The procedure to obtain carboxylic-functionalization is detailed in Rodriguez et al. [76], while amine 
functionalization is described in Rodriguez et al. [77]. A brief overview of the process is presented. Further details regarding proof of surface groups is found in $[78,79]$

\subsubsection{Carboxylic-Functionalization}

Initially, $2.5 \mathrm{~g}$ of as-received CNFs and $500 \mathrm{~mL}$ of nitric acid were mixed in a round bottomed flask using high-power sonication for $20 \mathrm{~min}(170 \mathrm{~W})$. This solution was then heated to $260{ }^{\circ} \mathrm{C}$ and stirred for $4 \mathrm{~h}$. The solution was then filtered through a $0.45 \mu \mathrm{m}$ pore filter paper, and rinsed using reverse osmosis water, acetone, and ultra pure deionized water until chemically neutral. The remaining O-CNFs were then dried overnight in a vacuum oven at $100{ }^{\circ} \mathrm{C}$. Finally, the O-CNFs were ground into a powdery form using a cryogenic grinder and stored in a desiccator.

\subsubsection{Amine-Functionalization}

Amine functionalization of CNFs was performed by first performing the steps for creating O-CNFs on as-received CNFs. Then, $5 \mathrm{~g}$ of O-CNFs, $80 \mathrm{~mL}$ of thionyl chloride, and 4 $\mathrm{mL}$ of dimethylformamide (DMF) were sonicated at $80 \mathrm{~W}$ for $20 \mathrm{~min}$. The solution was then heated to $65{ }^{\circ} \mathrm{C}$ and held for $24 \mathrm{~h}$. The solution was then distilled and the remaining CNFs were mixed with $100 \mathrm{~mL}$ of ethylenediamine and $5 \mathrm{~mL}$ of DMF. This mixture was heated to $100{ }^{\circ} \mathrm{C}$ and stirred for $48 \mathrm{~h}$. The mixture was then cooled to room temperature and diluted with ultrapure water before filtering through $0.45 \mu \mathrm{m}$ pore filter paper. The resulting A-CNFs were dried overnight in a vacuum oven at $60^{\circ} \mathrm{C}$, cryogenically ground, and stored in a desiccator.

\subsubsection{Electrophoretic Deposition of O-CNFs onto Single Carbon Fibers}

Electrophoretic deposition was performed by first dispersing $6.5 \mathrm{mg}$ of O-CNFs in 300 $\mathrm{ml}$ of ultrapure water using sonication (15 min at $140 \mathrm{~W}$ ). The mixture was then combined with an additional $800 \mathrm{ml}$ of ultrapure water in a clean electrophoretic tank resulting in an O-CNF to water concentration of $5.9 \mu \mathrm{g} / \mathrm{ml}$. This suspension was then lightly mixed by hand using a 
sterilized glass rod to ensure uniform dispersion within the tank. Individual carbon fibers were attached lengthwise to a metal frame using quick-dry adhesive. This frame was placed between two positioning holders for placement in the EPD tank. A diagram of the deposition setup is provided in Figure 13.

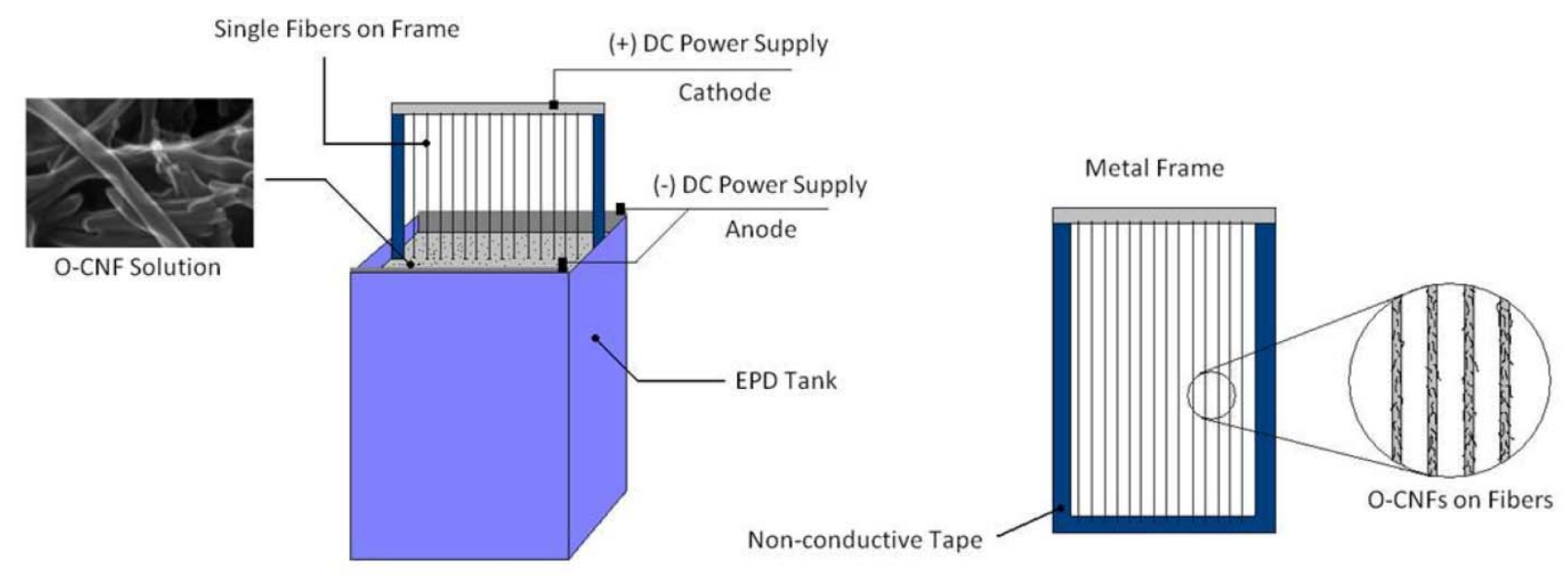

(a)

(b)

Figure 13: Single fiber EPD setup. Frame-mounted fibers being lowered into EPD tank (a); Fibers mounted on frame post deposition (b).

Once the frames were lowered into the tank containing the O-CNF solution, a $30 \mathrm{~V}$ field was applied at a distance of $60 \mathrm{~mm}$ from anode to cathode. Deposition was performed for $1 \mathrm{~min}$ on both sized and unsized fibers. In addition, samples for sized fibers undergoing 5 min of deposition were prepared. Further, the effects of EPD were investigated by exposing sized and unsized carbon fiber samples to the electric field without the presence of O-CNFs. After treatment in the EPD tank, the fibers were carefully removed from solution while maintaining the electric field to prevent the O-CNFs from being pulled from the fiber surface. The fibers were then dried in an oven at $100{ }^{\circ} \mathrm{C}$ for $1 \mathrm{~h}$. 
Deposition was verified using a FESEM. A single sized carbon fiber with O-CNFs deposited for 1 min is shown in Figure 14.

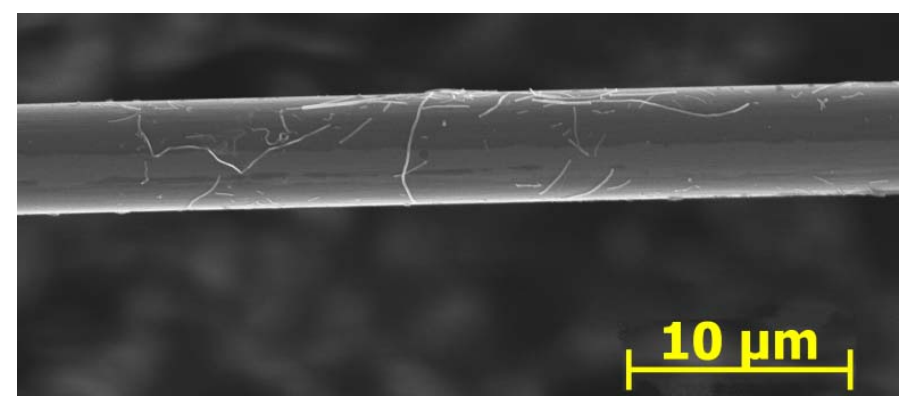

Figure 14: Sized carbon fibers with O-CNFs deposited.

\subsubsection{Electrophoretic Deposition of A-CNFs onto Single Carbon Fibers}

Individual sized carbon fibers were attached to a metal frame using adhesive. Initial ACNF EPD was performed by first dispersing $6.5 \mathrm{mg}$ of A-CNFs in $200 \mathrm{ml}$ of ultrapure water using sonication (20 min at $140 \mathrm{~W}$ ). The mixture was then combined with $900 \mathrm{ml}$ of ultrapure water in an electrophoretic tank resulting in an A-CNF to water concentration of $5.9 \mu \mathrm{g} / \mathrm{ml}$. The frames were then lowered into the tank containing the " $1 \mathrm{X}$ ” concentration A-CNF solution, and a $30 \mathrm{~V}$ field was applied at a distance of $60 \mathrm{~mm}$ between the electrodes. Deposition was performed for 1 min using the fibers as the anode.

Additional EPD was performed according to the same process above but with twice the concentration of A-CNFs $(2 \mathrm{X})$ in water $(11.8 \mu \mathrm{g} / \mathrm{ml})$. This increased the amount of A-CNF deposited on the fiber surface, but also increased the presence of agglomerates and A-CNF layering on these samples (Figure 15), as will be discussed later. In order to reduce the presence of the A-CNF clusters, a sequential sonication (SS) approach to gradually break up the nanoparticles was employed. First, $13 \mathrm{mg}$ of A-CNFs was sonicated in $50 \mathrm{ml}$ of ultrapure water 
$(140 \mathrm{~W})$ for $5 \mathrm{~min}$. This was followed by adding $50 \mathrm{ml}$ of ultrapure water every $5 \mathrm{~min}$ up until $20 \mathrm{~min}$ (200 $\mathrm{ml}$ total) of sonication time had elapsed. The mixture was then combined with 900 $\mathrm{ml}$ of ultrapure water in the tank and one-stage anodic deposition was performed as previously noted to produce the $2 \mathrm{X}$-SS fiber samples.
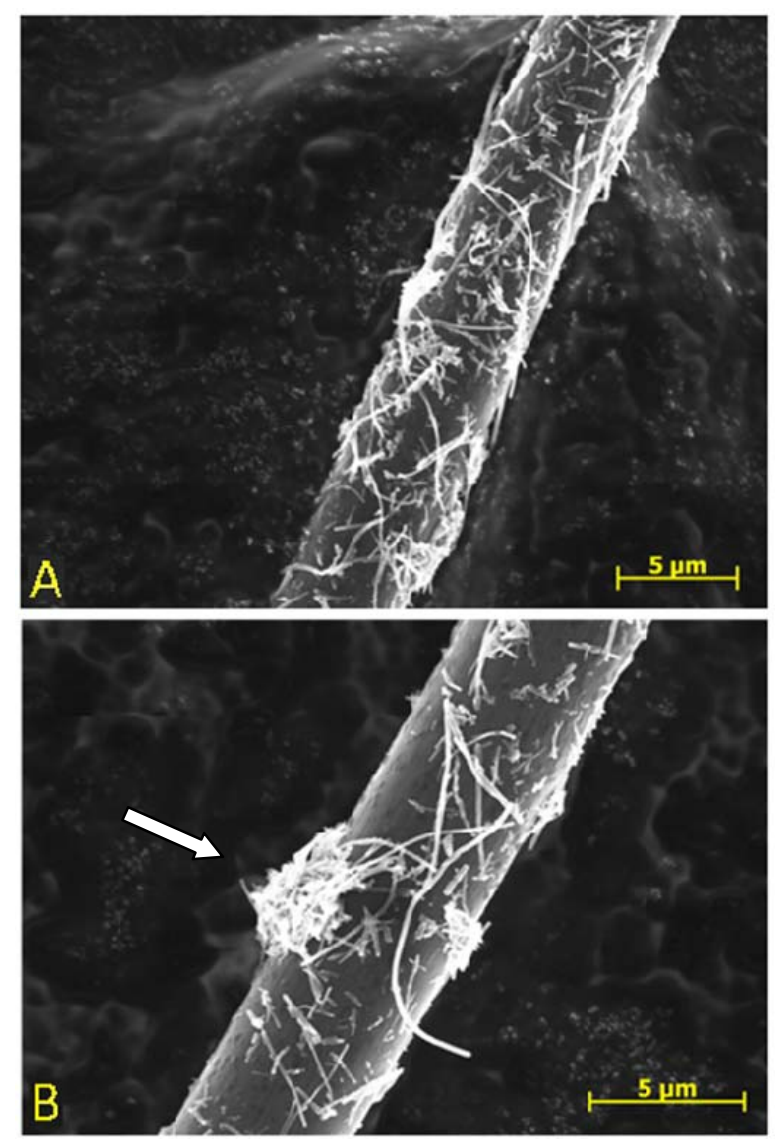

Figure 15: Single carbon fiber after one-stage $2 \mathrm{X}$ concentration EPD. Layering shown in A; agglomerate indicated by white arrow in $\mathrm{B}$.

A two-stage A-CNF EPD approach was performed by preparing a 2X-SS solution and lowering the mounted fibers into the tank. In the first stage, the electric field was applied at $30 \mathrm{~V}$ with the fibers acting as the cathode. After $1 \mathrm{~min}$, the field was switched so that the fibers acted as the anode for an additional minute; these fibers are denoted as "2S-2X-SS." 
After treatment in the EPD tank, all fibers were carefully removed from solution while maintaining the electric field to prevent the CNFs from being pulled from the fiber surface. The fibers were then dried in an oven at $100{ }^{\circ} \mathrm{C}$ for $1 \mathrm{~h}$.

\subsubsection{Tensile Strength Testing of Single Carbon Fibers}

To build the sample population for Weibull analysis, approximately 40 samples of each fiber type were individually tested for tensile strength using a Linkam TST 350 stage with $20 \mathrm{~N}$ load cell and $1 \mathrm{mN}$ resolution. Fibers were glued to stiff paper frames to aid with transportation and alignment; the frames were gripped by the testing stage and cut prior to testing. The fiber diameter was determined using an optical microscope and performing at least five measurements per fiber. Testing was conducted per ASTM standards C1239 and C1557. The gage length was $15 \mathrm{~mm}$.

\subsubsection{Fragmentation Specimens}

Fibers were placed into notches in the silicone mold and adhesive was used to hold the fiber ends in place during resin addition. The resin system consisted of Epon 828 epoxy from Hexion Specialty Chemicals and 4,4' -methylenedianiline 97\% mixed in a 100:26.7 ratio by weight. This system provided the increased matrix strain to failure needed for achieving fragment saturation. After mixing and degassing in a planetary centrifugal vacuum mixer at $2000 \mathrm{rpm}$ for four, 5 min cycles, the resin was added to the mold. The specimens were cured at $60{ }^{\circ} \mathrm{C}$ for $8 \mathrm{~h}$ followed by $120{ }^{\circ} \mathrm{C}$ for $8 \mathrm{~h}$ before cooling to room temperature. Final gage dimensions were $1 \mathrm{~mm}$ thick, $2 \mathrm{~mm}$ wide, and $12 \mathrm{~mm}$ long; all samples were lightly sanded to remove burs and edge irregularity.

\subsubsection{Single Fiber Fragmentation Test}

As noted in Chapter 1, the single fiber fragmentation test uses tensile stress to investigate stress transfer from the matrix to the fiber through shear stress at the interface. Because the 
matrix is considerably more ductile than the carbon fiber, it will deform to a greater degree. Depending on the interface strength, a fiber segment will break under increasing strain while its length is greater than a critical fiber length, $l c$. The fragmentation process continues until saturation, which is the point at which no further breaks occur; this represents the stress transfer limit between the matrix, interface, and embedded fiber. The term $l c$ is considered to be characterized by a normal distribution that is determined from the average fragment length at saturation using the following formula [13]:

$$
\text { Ic }=\left(\frac{4}{3}\right) * l_{\text {avg }}
$$

where $l_{\text {avg }}$ is the average fragment length. Kelly and Tyson [20] assumed a constant state of shear stress at the interface, and from this assumption the IFSS was calculated:

$$
\tau_{\text {IFSS }}=\left(\frac{\sigma_{f}}{2}\right) *\left(\frac{d}{l c}\right)
$$

where $\tau_{\text {IFSS }}$ is interfacial shear strength, $d$ is the fiber diameter, $l c$ the fiber critical length, and $\sigma_{f}$ is the calculated fiber strength at the critical length. Figure 16 provides a schematic of the fiber fragmentation test. 


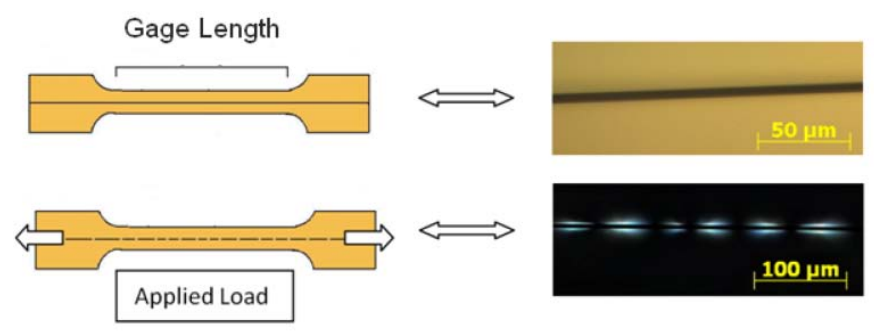

Figure 16: Diagram of fiber fragmentation.

The dogbone-shaped specimens were aligned on the tensile stage and the system was fitted with a $200 \mathrm{~N}$ load cell having $10 \mathrm{mN}$ resolution. At least five specimens for each fiber condition were tested. Fiber breaks were counted at each load increment (5 N) using an optical microscope affixed with polarized light. The gage length was divided by the number of fragments to determine the average fragment length, and saturation was achieved for all samples used in calculation.

\subsubsection{Weibull Analysis of Single Fiber Tensile Failures}

Due to internal and surface defects, carbon fiber tensile strength is typically influenced by a size effect. Thus, tensile strength in carbon fibers increases as the length of the fiber decreases [21, 22, 80]. Because it is difficult to measure the fiber tensile strength at the critical lengthoften on the order of hundreds of microns - a weakest link scaling function based on Weibull analysis was used for each fiber type:

$$
\sigma_{f}=\sigma_{u l t} *\left(\frac{l c}{l o}\right)^{-\frac{\alpha}{\rho}}
$$

where $\alpha=0<x \leq 1$, lo is the tensile test fiber length, $\sigma_{f}$ is the mean critical length tensile strength, $\sigma_{u l t}$ is the Weibull scale parameter, and $\rho$ is the Weibull shape parameter. The term $\alpha$ is a correlation factor determined from tensile testing of fibers with different lengths and is related to fiber properties along the fiber length. Beyerlein and Phoenix [21] showed that for 
longer lengths of Hercules AS4 carbon fibers ( $200 \mathrm{~mm}$ gauge length), $\alpha \sim 0.6$, while Otani et al. [22] determined $\alpha \sim 1$ for Hercules IM6 fibers at shorter lengths ( $x \leq 30 \mathrm{~mm})$. In light of these findings, $\alpha$ was assumed to be unity due to the use of similar materials and testing involving gage lengths below $30 \mathrm{~mm}$.

Weibull analysis was performed by plotting the Weibull cumulative probability of fiber tensile failure $(\ln (-\ln (1-P)))$ against the log of fiber strength on a log-log scale and determining the shape and scale parameters. The two-parameter Weibull plot for O-CNF and cathode-based EPD fibers is provided later on in Chapter 4, in Figure 17. The probability of fiber failure $(P)$ was estimated for each fiber using its median rank approximation $(m)$ as given in ASTM C1239:

$$
m=\left(\frac{i-0.5}{n}\right)
$$

with $i$ being the ascending strength rank and $n$ the sample population per fiber treatment. 


\section{CHAPTER 4}

\section{RESULTS AND DISCUSSION}

\subsection{Effects of Electrophoretically Deposited O-CNFs on the Interface of Single Carbon Fibers Embedded in Epoxy Matrix}

This section presents and discusses the results for fibers acting as the cathode during onestage EPD of O-CNFs. In order to determine the effect of the electric field on the fibers during the deposition process, single fibers acting as the cathode in ultrapure water without the presence of O-CNFs were also tested.

Table 3: Fiber Parameters

\begin{tabular}{lcccc}
\hline Surface Condition & Diameter $(\mu \mathrm{m})$ & W. Shape & W. Scale $(\mathrm{MPa})$ & \# Samples \\
\hline Sized & 5.22 & 4.60 & 4517 & 48 \\
Sized Electric Field 1 min & 5.19 & 2.59 & 2618 & 40 \\
Sized w/ O-CNFs-EPD 1 min & 5.19 & 2.41 & 3616 & 47 \\
Sized w/ O-CNFs-EPD 5 min & 5.18 & 2.77 & 2497 & 44 \\
Unsized & 5.19 & 3.62 & 4007 & 45 \\
Unsized Electric Field 1 min & 5.19 & 2.76 & 3855 & 40 \\
Unsized w/ O-CNFs-EPD 1 min & 5.16 & 3.40 & 3779 & 40 \\
\hline
\end{tabular}

As indicated in Table 3, the process to remove fiber sizing slightly decreased fiber tensile strength (lower scale parameter) and increased sample variability (lower shape parameter). While exposing sized fibers to the electric field decreased tensile strength, unsized fibers appeared to be less affected. Additional processing steps such as EPD and drying may damage the fiber surface thereby decreasing fiber strength; however, sized fiber samples undergoing 1 and 5 min O-CNF deposition were both exposed to the same drying process but exhibited drastically different failure strengths, which suggests drying is not the cause. Unsized fiber samples exposed to the electric field for 1 min and undergoing O-CNF deposition both showed a small decrease in failure strength by comparison. 


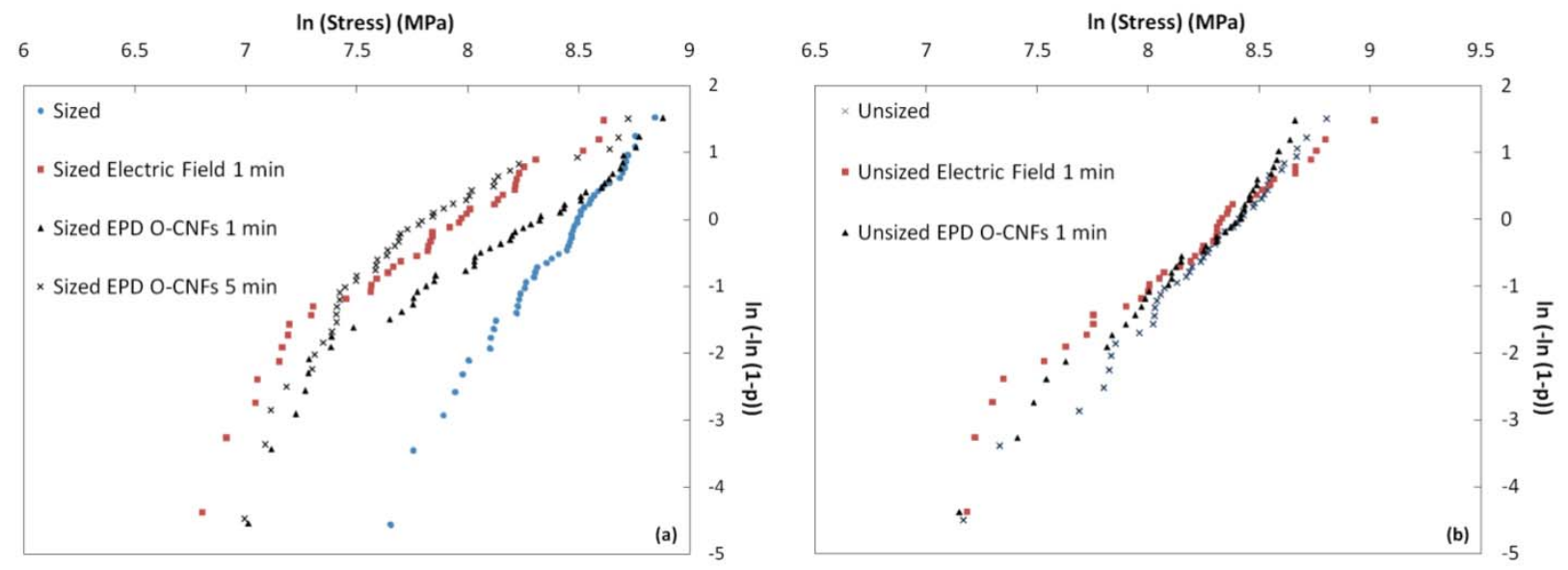

Figure 17: Two-parameter Weibull plot for sized, unsized, O-CNF deposited, and electric field fibers.

Depicted in Figure 17 (a), the sized fibers exposed to an electric field for 1 min exhibited a similar Weibull behavior as the fibers undergoing O-CNF deposition for $5 \mathrm{~min}$. Sized fibers with O-CNF deposited for 1 min relate similar failure strengths to both the base sized fibers and fibers exposed to the electric field. SEM review of the materials (to be addressed later) indicated that the non-conductive epoxy sizing is affected by exposure to the electric field in the presence of water and that this may decrease the fiber tensile strength. The addition of O-CNFs to the surface appears to limit this decrease for the 1 min deposition cycle, but any mitigating effects of O-CNF deposition no longer appear to be effective when the time is increased to $5 \mathrm{~min}$. The unsized fiber samples (Figure 17 (b)), indicate increased failure variability with exposure to the electric field in the presence of water. However, the mean failure strength is not nearly as affected as in the case of the sized fibers, which further suggests a link between sizing, electric field, and failure strength.

\subsubsection{Single Fiber Fragmentation}

All sample types were tested and the break behavior is illustrated in Figure 18. As indicated, at least five samples for each fiber preparation reached saturation. Compared to the 
sized fibers, the unsized fiber samples tended to start breaking at higher applied tensile stress and exhibited fewer overall fragments. Coupled with the failure strength data, this behavior implies that stress was not transferred as effectively. The O-CNF deposited unsized fibers produced moderately more breaks than either sized or unsized base fiber samples while the O-CNF deposited sized fibers provided samples with the most breaks.

A trend was noted in the fragmentation behavior of O-CNF deposited sized samples. In general, samples that had greater O-CNF deposition uniformity and close wrapping to the carbon fiber contained more breaks. Eight samples were included for the 5 min sized fiber deposition analysis due to these samples having a large range of fragmentation response (Figure 18 (a)). It was noted that the samples with the lowest number of fragments contained uneven and agglomerated deposition while the highest numbering samples were defined by highly uniform O-CNF deposition closely bound to the fiber surface and no agglomerates. The above trend (as well as varying fiber strength between samples) may explain the observed range of fragments at saturation. Each sized sample with 1 min of O-CNF deposition had a high level of deposition uniformity which lead to a more homogeneous interface than the 5 min deposition samples. This is reflected in the lower variability of fragmentation response shown in Figure 18 (a). 


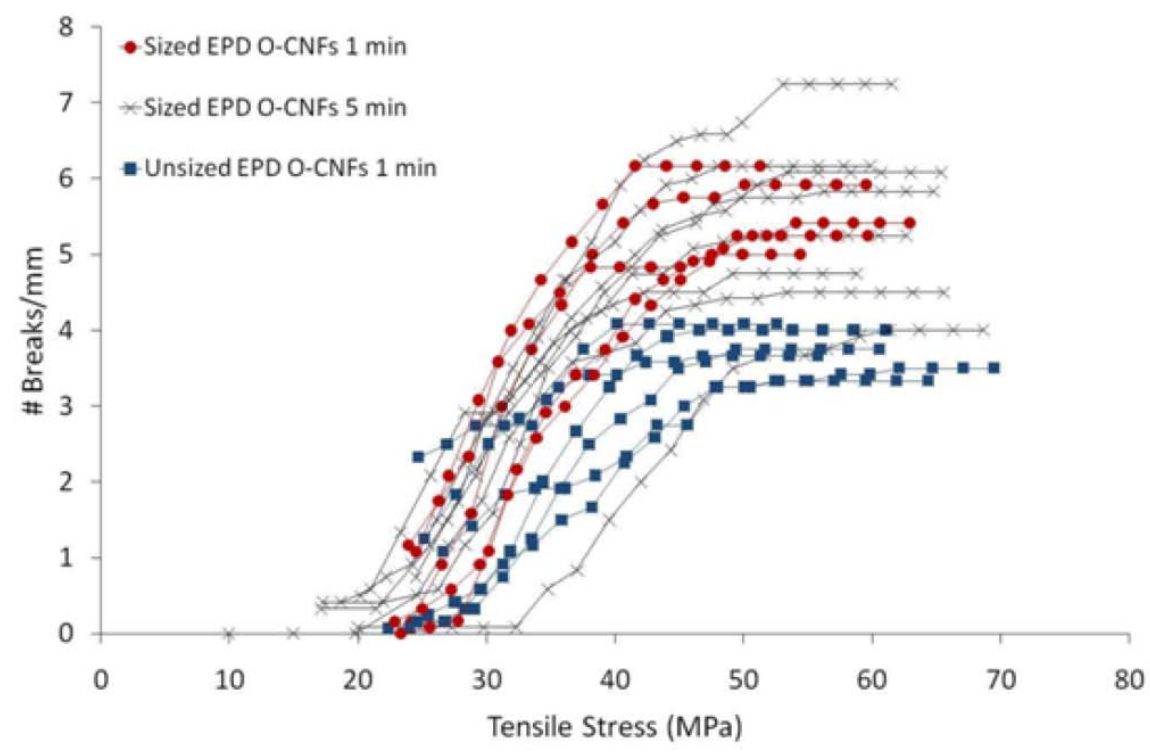

(a)

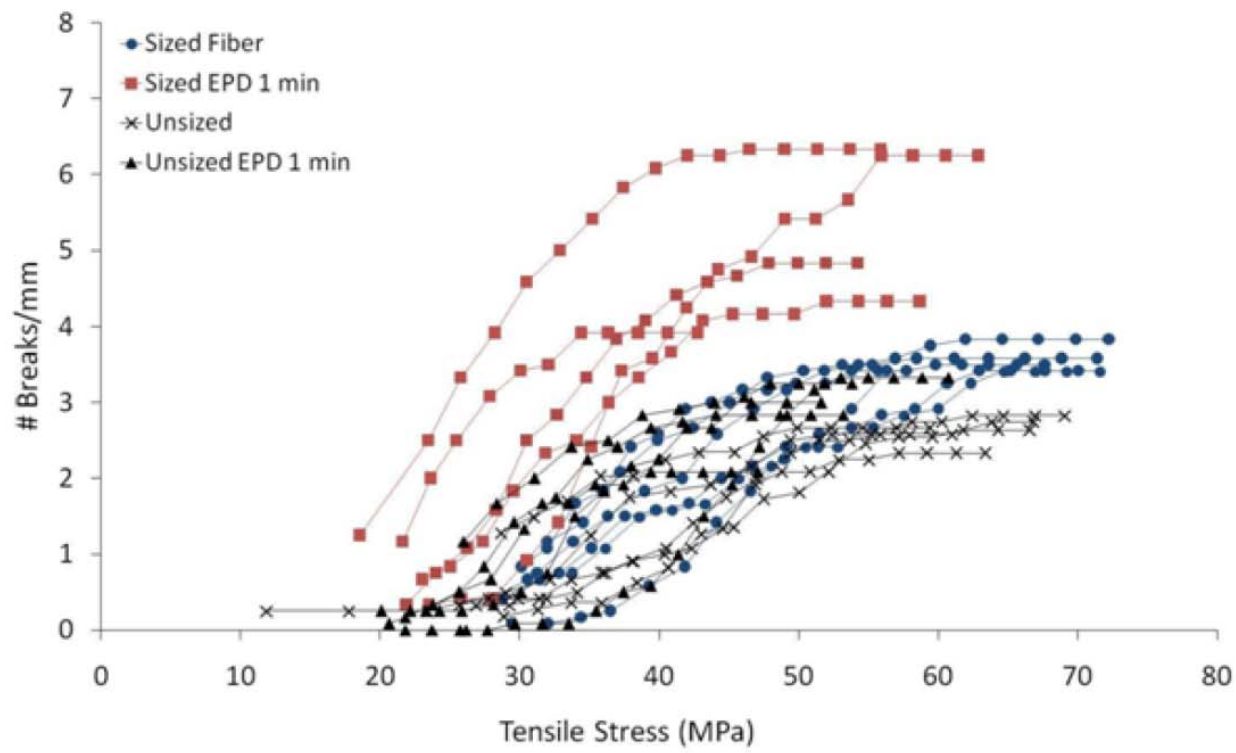

(b)

Figure 18: Break behavior for each fiber sample type.

Another trend was noted by comparing the break behavior between plain samples (Figure 18 (b)) and those with O-CNF deposition. Fibers with deposition tended to produce more fragments at lower applied stress and reached saturation more rapidly than those without deposited O-CNFs. Although fiber strength varies by surface treatment (Table 3), the incidence of saturation at lower applied stress may indicate increased interface stiffness. In Figure 18 (b), the fragment behavior of the sized samples exposed to an electric field for $1 \mathrm{~min}$ is scattered and 
the samples do not appear to follow any trend with respect to each other. The lack of a uniform response could be due to a non-homogeneous interface created by damage to the carbon fiber surface.

\subsubsection{Interfacial Shear Strength}

Using the critical fiber length determined from the fragmentation data and Eq. (1), IFSS was calculated using equation (2). The results are depicted in Figure 19 and Figure 20.

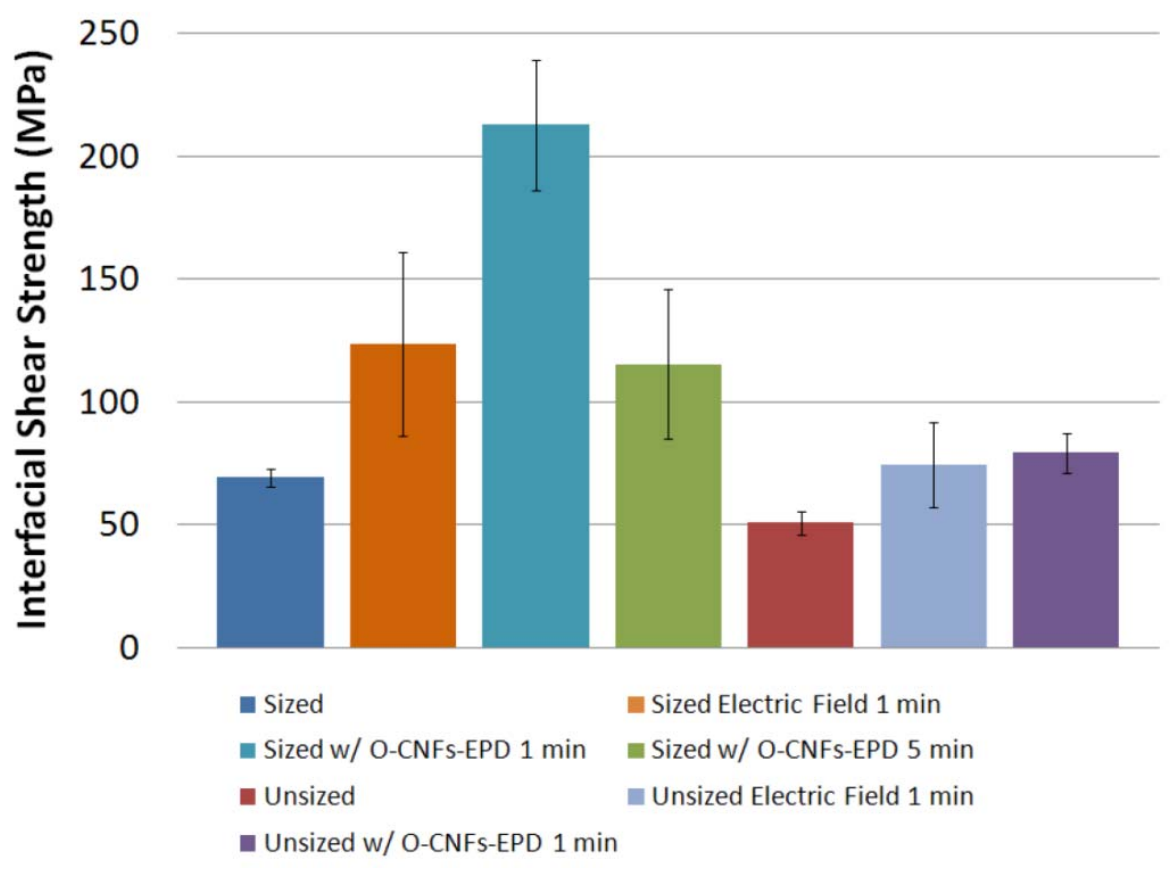

Figure 19: Interfacial shear strength for each sample type. Error bars represent one standard deviation. 


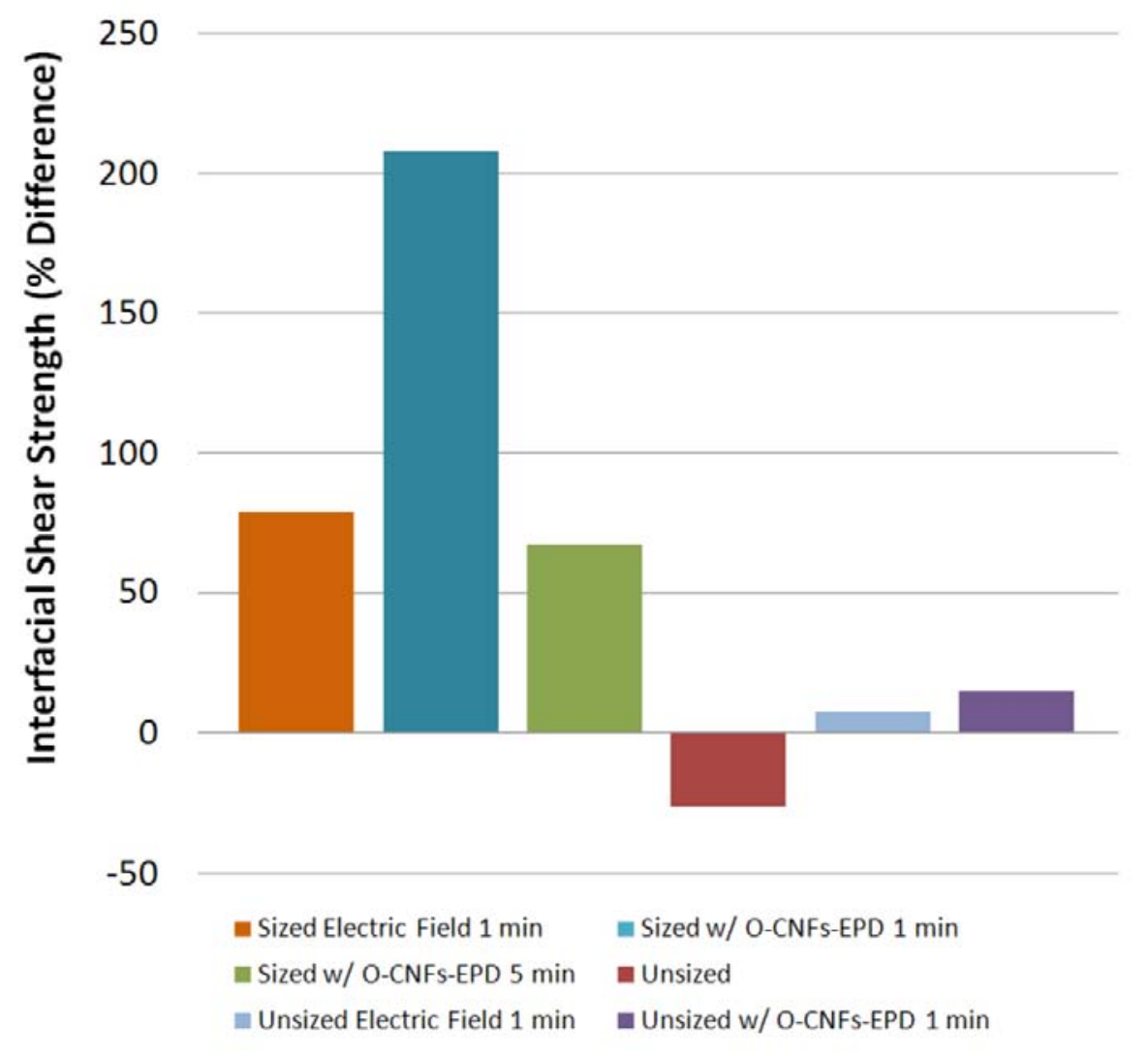

Figure 20: Percent difference compared to base sized fibers.

Removal of the fiber sizing decreased IFSS by approximately $27 \%$. The addition of OCNFs to the unsized fiber surface led to an increase of $15 \%$ compared to the base sized fibers and a 56\% increase compared to the unsized fiber samples. The O-CNF deposited sized fibers provided IFSS increases of $207.6 \%$ for the 1 min field duration, and $66.9 \%$ for the $5 \mathrm{~min}$ duration compared to the base sized fiber samples. Exposing sized fibers to the electric field for 1 min led to an increase of $79 \%$, but significantly increased the coefficient of variation (COV) from $5.5 \%$ to $30.5 \%$ of the respective sample mean. Unsized fibers undergoing the same treatment provided increases of $7.7 \%$ and $46 \%$ compared to the base sized fiber and unsized fiber samples, respectively. These increases may be due to further removal of any residual fiber sizing or increased surface roughening. The COV for sized, unsized, and O-CNF deposited unsized 
fibers was relatively low which could indicate uniform surface conditions for each respective surface treatment.

Zhang et al. [17] creatively used EPD to deposit multi-walled CNTs on the surface of glass fibers and achieve a 30\% IFSS increase in single fiber epoxy composites. Park et al. [18] employed the fragmentation test to investigate the interface created by using EPD to deposit exfoliated graphene nanoplatelets on the surface of carbon fibers in an epoxy matrix (Epon 828). By adjusting field potential, these researchers characterized how changing the field affects IFSS; the IFSS increased from a base value of 50.9-55.5 MPa when applying a $50 \mathrm{~V}$ field for $5 \mathrm{~min}$. Additionally, a 15\% increase in IFSS was achieved by Thostenson et al. [9] who investigated the growth of CNTs on the surface of unsized carbon fibers using CVD. In comparison to these and the previously mentioned interface investigations, it is apparent that EPD holds much promise for enhancing the fiber-matrix interface.

\subsubsection{Surface Characteristics and Break Behavior}

As determined by the fragmentation response and calculated IFFS values, the fiber surface was affected differently by each surface treatment. Using an FESEM, the fiber surfaces were reviewed and representative images are provided in Figure 21 and Figure 22. Sized fibers were defined by smooth surfaces with few flaws. Unsized fiber surfaces, however, appeared to be damaged and partially covered with what may be remaining epoxy sizing. Sized fibers exposed to an electric field for 1 min indicated that the sizing had begun to peel away from the carbon fiber in some areas (arrows, Figure 21 (c)), while unsized fibers exposed to the same conditions did not show increased visual damage over the base unsized fibers. In Figure 21 (e), a sized fiber undergoing 1 min of O-CNF deposition is shown. The fiber surface is covered with short and long O-CNFs that are positioned closely to the fiber surface. In general, long O-CNFs were oriented lengthwise along the axis of the carbon fiber while short O-CNFs were more 
randomly oriented. This alignment behavior is attributed to the surface energy of the liquid medium acting on the carbon fibers during removal from the EPD tank after deposition. The occurrence of sizing peeling from the carbon fiber surface was also not readily apparent. The unsized fibers with deposited O-CNFs had generally less deposition than the sized samples. This may infer that the O-CNFs did not adhere as well to the unsized carbon fiber surface as the sized fiber surface. 

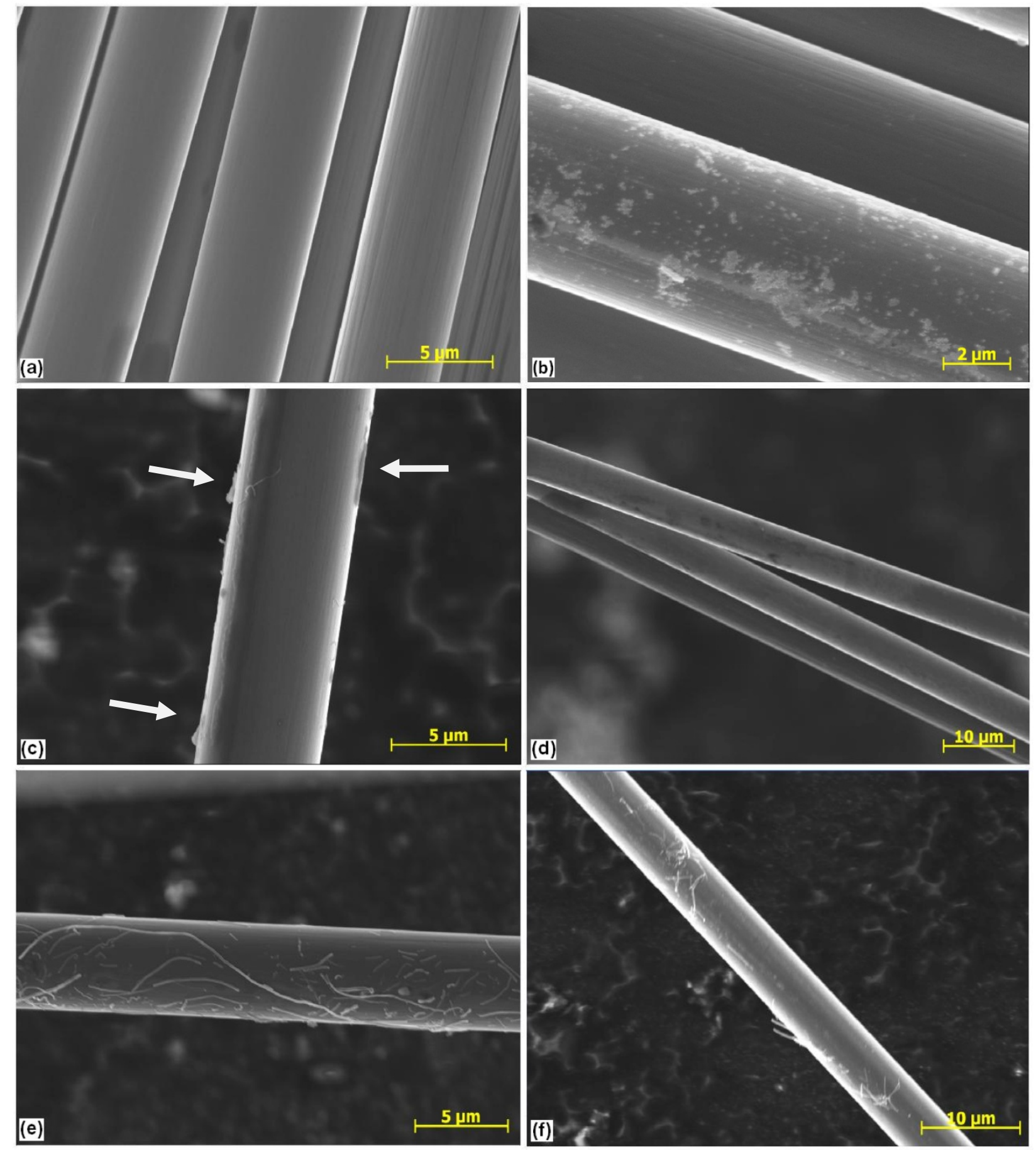

Figure 21: Sized fibers (a), unsized fibers (b), sized fiber exposed to 1 min electric field (c), unsized fibers exposed to 1 min electric field (d), sized fiber with 1 min O-CNF deposition (e), and unsized fiber with 1 min O-CNF deposition (f).

As indicated in Figure 18 (a), sized fibers undergoing 5 min of O-CNF deposition exhibited the largest variation in break behavior. Inspection of these fiber surfaces indicated a 
clear difference in deposition quality between fibers (Figure 22). As illustrated, three fiber surfaces were prevalent among those studied: an irregular surface defined by dense O-CNF deposition and degraded sizing, a surface with intermediate deposition and degraded sizing, and a surface containing low deposition and degraded sizing. Reviewing optical microscope images of the associated fragmentation samples indicated that more breaks were present in samples having surfaces similar to the carbon fiber in the top image of Figure 22, while the least breaks were present for fibers with surfaces similar to the fiber in the bottom image of Figure 22. In the top image, the rough surface is covered by O-CNFs that have been surrounded by the fiber sizing. The sizing/O-CNFs mixture has begun to agglomerate, leaving bare patches on the carbon fiber surface which are clearly seen in Figure 23. This creates an irregular surface condition that increases surface area for stress transfer in the fragmentation samples, but may also be the cause of the decreased carbon fiber tensile strength indicated in Table 3. Experiments have suggested that the epoxy sizing may be slightly miscible in water, which could potentially explain how the O-CNFs and sizing become intertwined. The central image shows a fiber surface similar to that of those undergoing 1 min of O-CNF deposition, while the bottom image shows a fiber with considerably less O-CNF deposition. The presence of white spheres (100$500 \mathrm{~nm}$ diameter) on the surface of all sized fibers undergoing 5 min of O-CNF deposition was noted, and almost every fiber could be characterized by these three categories. 


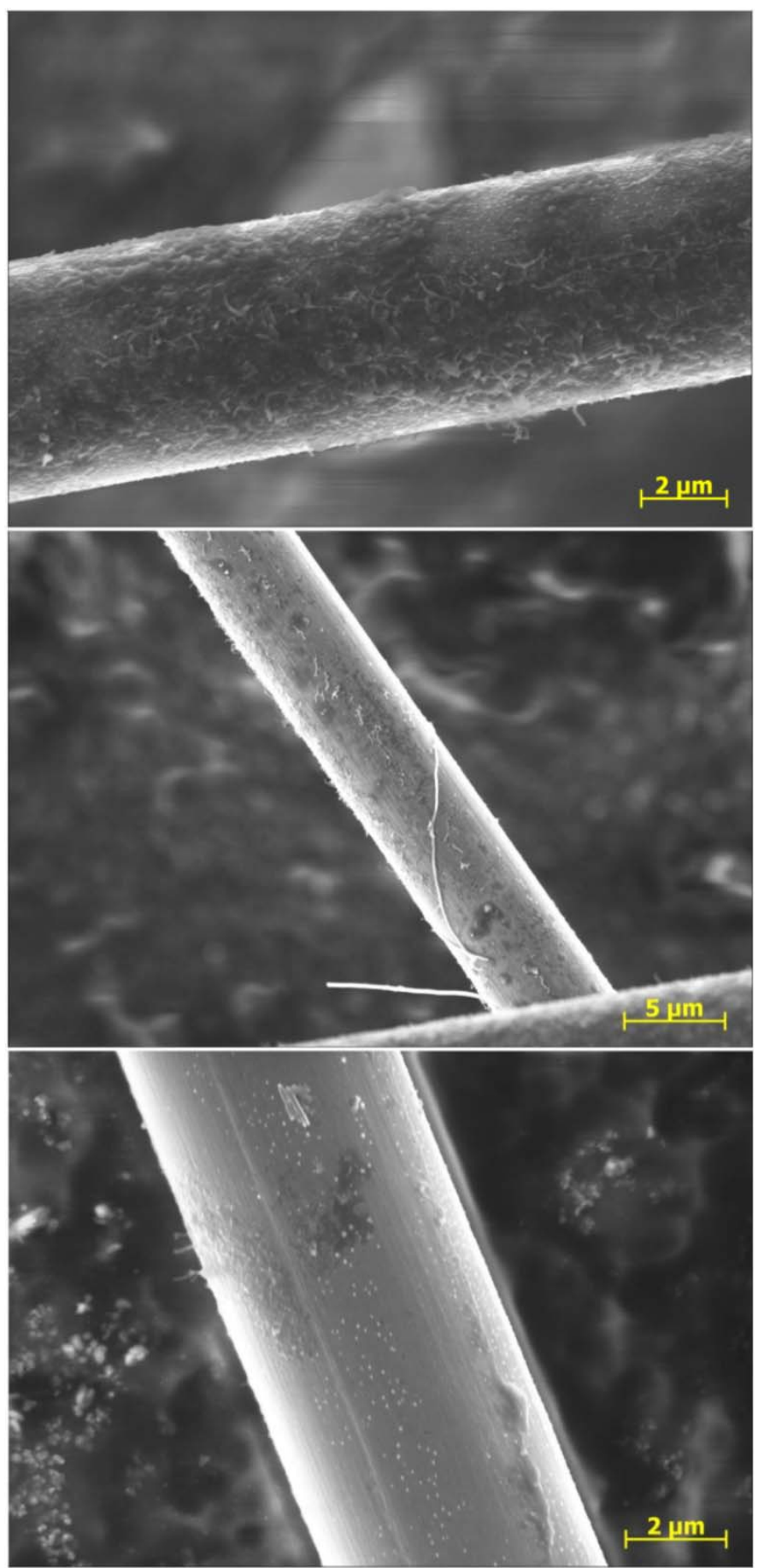

Figure 22: Sized fibers with 5 min O-CNF deposition. Irregular surface with high level of deposition (top), intermediate level of deposition (middle), and low level of deposition (bottom). 


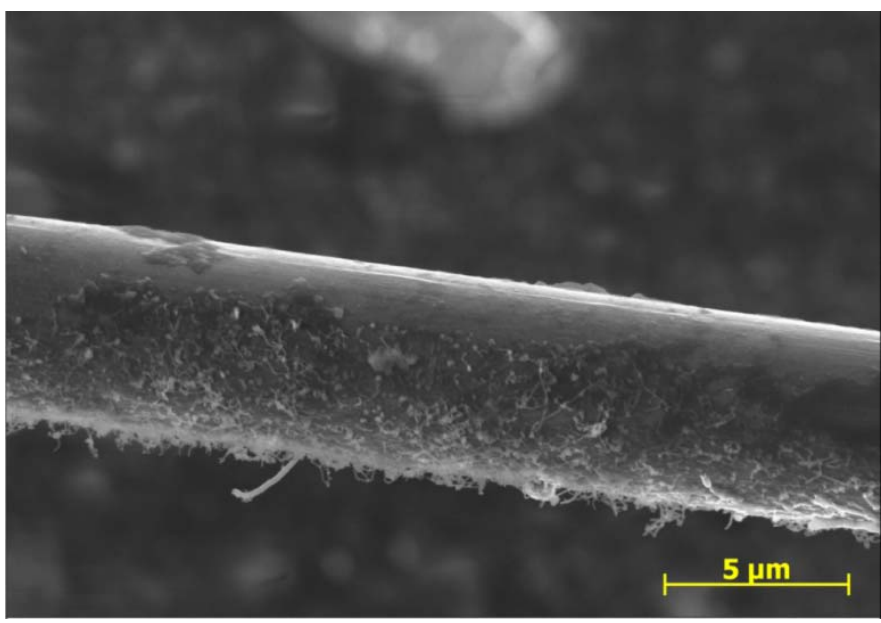

Figure 23: Exposed carbon fiber surface due to agglomeration of O-CNFs and deterioration of the epoxy sizing.

A review of fragmentation samples using optical microscopy revealed differences in fiber break behavior. Close up images of breaks are shown in Figure 24. Sized fiber breaks were typically large and extended into the matrix (Figure 24 (a)). It is believed that the sizing helped to uniformly distribute the load from the matrix to the fiber. The interaction between fiber and matrix appears to be strong, since the matrix was damaged by the fiber break and no delamination was present.

Unsized fiber samples displayed regions of delamination surrounding the fiber breaks. The breaks did not extend into the matrix; instead, debonding is present at the fiber-matrix interface (Figure 24 (b)). It was observed that this debonding primarily occurred at the moment of fracture and that local stress relaxation of the fiber as well as continued debonding under increasing load are at fault. As the stress increases, the surface area lost to debonding increases and this decreased the surface area available for stress transfer from the matrix. 


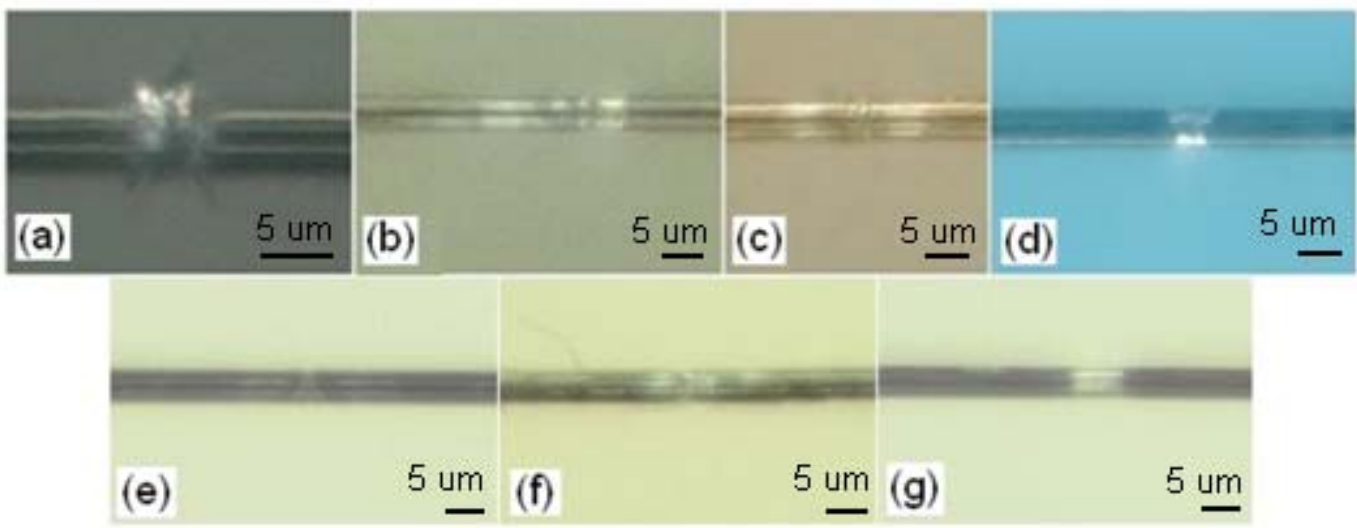

Figure 24: Fiber breaks for sized fiber (a), unsized fiber (b), sized fiber with 5 min O-CNF deposition (c), unsized fiber with 1 min O-CNF deposition (d), sized fiber exposed to 1 min electric field (e), sized fiber with 1 min O-CNF deposition (f), unsized fiber exposed to $1 \mathrm{~min}$ electric field (g).

In contrast to the sized fibers without deposition, the sized fibers with O-CNF deposited for $1 \mathrm{~min}$ and 5 min (Figure 24 (c) and (f), respectively) did not display breaks that extended into the matrix. This may indicate that the O-CNFs act as reinforcement to the matrix during a break, which would imply a closer association with the matrix than the carbon fiber surface. Slight delamination was also present, although to a lesser extent in the 1 min cycle samples.

Review of the unsized fibers with O-CNFs indicated small and sharp breaks that were localized to the fiber only (Figure 24 (d)). The matrix remained largely unaffected and only a limited amount of delamination occurred. This could be due to increased interaction between the deposited O-CNFs, the matrix, and the fiber that results from removal of the epoxy sizing and the presence of functional groups on the O-CNFs. The sized fibers exposed to the electric field for 1 min (Figure 24 (e)) produced small and angled breaks that did not extend into the matrix. Delamination was not as apparent as those in (c), and the fiber does not appear to pull away from the break zone. In contrast, the unsized fibers exposed to the electric field produced mainly straight breaks and the fiber pulled away from the break zone (g). Further, debonding was 
typically not present which may indicate that fiber relaxation was more localized than in the other samples.

Finally, the addition of O-CNFs to the surface of sized and unsized carbon fibers led to increases in IFSS. The sized fibers undergoing deposition for $1 \mathrm{~min}$ had surfaces characterized by closely bound long and short O-CNFs that were partially aligned in the axial direction. This increased the carbon fiber surface area available for enhanced interaction and mechanical interlocking with the matrix. The O-CNFs reinforced the interface in the plane of stress transfer and the epoxy sizing appears to enhance O-CNF addition by aiding in the stress distribution along the carbon fiber length. Extending the deposition time to 5 min visibly increased the sized fiber surface roughness due to degradation of the fiber sizing and the agglomeration of deposited O-CNFs. Exposing sized fibers to the electric field for $1 \mathrm{~min}$ also appeared to degrade the surface; the IFSS increase is attributed to surface roughness. 


\subsection{Effects of Electrophoretically Deposited A-CNFs on the Interface of Single Carbon Fibers Embedded in Epoxy Matrix}

\subsubsection{Tensile Strength and Weibull Parameters}

The parameters for Weibull analysis are listed in Table 4. In general, samples acting as the anode during one-stage deposition were not adversely affected by the treatment process. Previously, Schaefer et al. [81] determined the one-stage Weibull parameters for several fiber samples involving one-stage cathodic EPD; these values are included in the table for clear comparison. Also, a trial population of O-CNF deposited tensile samples was prepared and tested. While fewer samples were included for parameter estimation, the shape and scale parameters are considered valid due to similarity with those previously determined. Fibers undergoing two-stage A-CNF deposition exhibited a similar average tensile strength as the onestage O-CNF deposited fibers, and all sample preparations using sized carbon fibers as the cathode during one-stage or two-stage EPD, or in water had a lower average tensile failure strength than the base fiber. 
Table 4: Weibull Parameters

\begin{tabular}{lccc}
\hline Surface Condition & W. Shape & W. Scale (MPa) & \# Samples \\
\hline Sized Base Fiber & 4.27 & 4429 & 41 \\
A-CNF EPD 1X & 3.15 & 4384 & 43 \\
A-CNF EPD 2X & 3.09 & 4531 & 44 \\
A-CNF EPD 2X-SS & 3.27 & 4826 & 44 \\
A-CNF EPD 2S-2X-SS & 2.47 & 3432 & 38 \\
O-CNF EPD 1X & 2.23 & 3423 & 23 \\
Anode in Water & 3.25 & 5198 & 48 \\
Cathode in Water* & 2.59 & 2618 & 40 \\
\hline
\end{tabular}

* Schaefer et al. [81]

\subsubsection{Interfacial Shear Strength}

Initially, fibers underwent 1 min of $1 \mathrm{X}$ A-CNF deposition acting as the anode (Figure 25A); these samples provided an IFSS increase of $7.6 \%$ over the base fibers while maintaining a low coefficient of variation. Under the optical microscope, the surface of these fibers appeared to be smooth which inferred that any potential deposition was beyond optical magnification. Based on previous work with O-CNFs, effective deposition was typically verifiable using optical microscopy; accordingly, it was desired to achieve a surface condition with increased deposition, wherein, the A-CNFs would be visible as a rough layer on the carbon fiber surface. The IFSS for each fiber treatment is depicted in Figure 26 and Figure 27. The base value of $65.1 \mathrm{MPa}$ for the resin system is similar to the value of 69.2 MPa achieved by Schaefer et al. [81] and the value of 65.3 MPa determined by Umesh and Bernard [82], who used the microbond approach. 


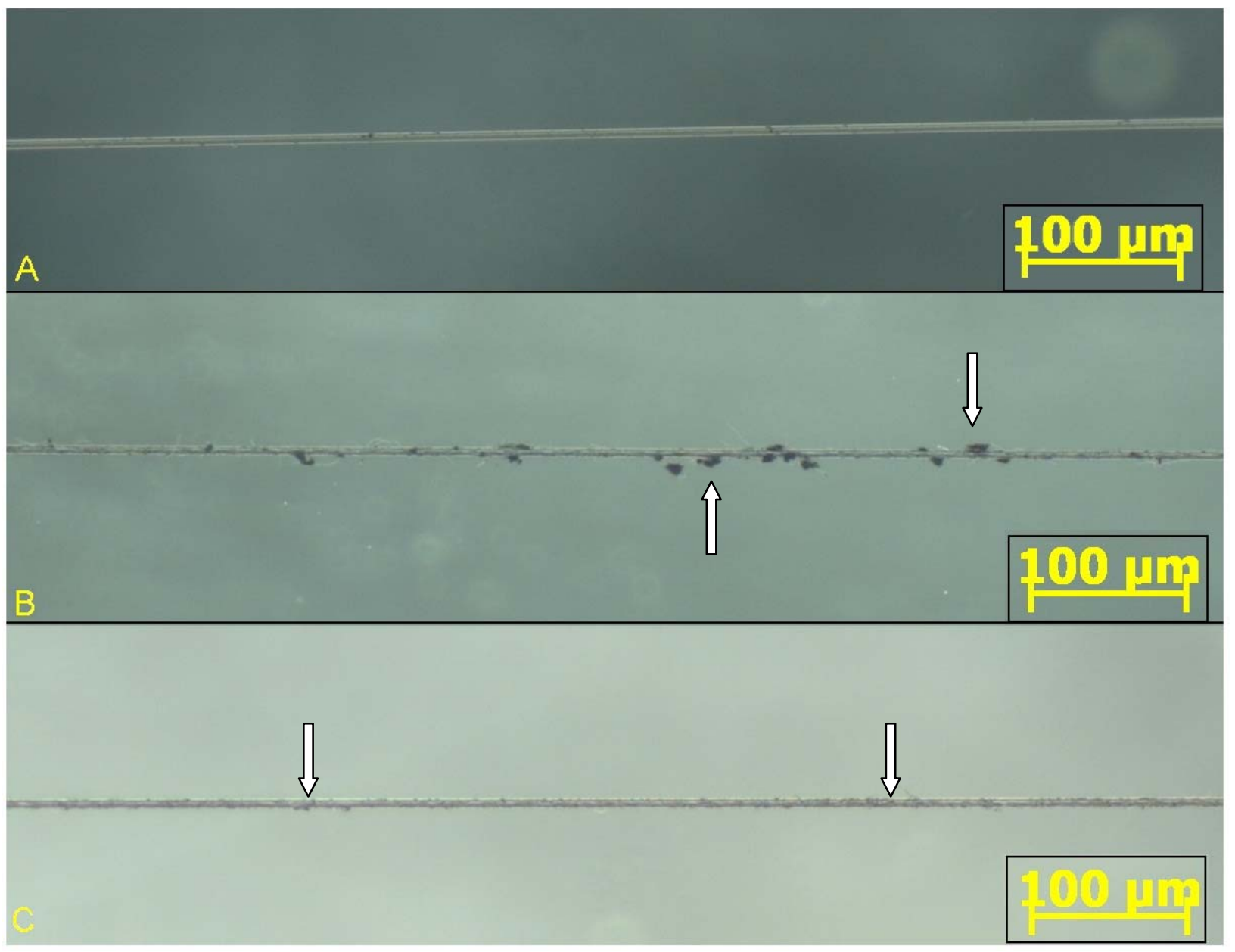

Figure 25: Single carbon fibers representing $1 \mathrm{X}$ concentration (A), $2 \mathrm{X}$ concentration (B), and 2X-SS (C) one-stage anode EPD approaches. In (B), arrows indicate agglomerates, while in (C) they indicate areas of increased surface disorder.

Previously, Rodriguez [79] assessed the movement of CNFs through water by using laser doppler velocimetry to determine the electrophoretic mobility (EM) of O-CNFs and A-CNFs in water. Higher EM values indicate that a particle will move faster during EPD which is a parameter affecting deposition rate. For the range of $\mathrm{pH}$ values studied, O-CNFs were negatively charged in water, while A-CNFs were positively charged between $2 \mathrm{pH}$ and approximately $7.5 \mathrm{pH}$. Thus, in the presence of a DC electric field, O-CNFs will travel towards the positive electrode while A-CNFs will travel towards a negative electrode for the specified $\mathrm{pH}$ range. For the deposition experiments in the present work, the $\mathrm{pH}$ for O-CNF solutions was 
4.8 and the $\mathrm{pH}$ for A-CNF solutions was $\sim 5.5$ with EM values of -4.6 and $2.8 \frac{\mu m \cdot \mathrm{cm}}{\mathrm{V} \cdot \mathrm{s}}$, respectively. Thus, the absolute value of O-CNF EM is approximately 2 times that of A-CNF, indicating that O-CNFs travel approximately twice as quickly as A-CNFs in water for the given $\mathrm{pH}$ values. Therefore, twice as many O-CNFs may be deposited as A-CNFs during a given time period. This disparity in deposition was generally noticed when viewing the tensile and fragmentation samples made from these nanoparticles at the same concentration.

In order to achieve increased A-CNF deposition, the concentration of the solution was increased to twice the original amount $(11.8 \mu \mathrm{g} / \mathrm{ml})$ to ensure significant deposition of A-CNF on the fiber surface. The result was an interface defined in some areas by closely layered A-CNFs that extended along the fiber surface (Figure 15). In other locations, the $2 \mathrm{X}$ surface was defined by the presence of large agglomerates in some areas (Figure 25B).

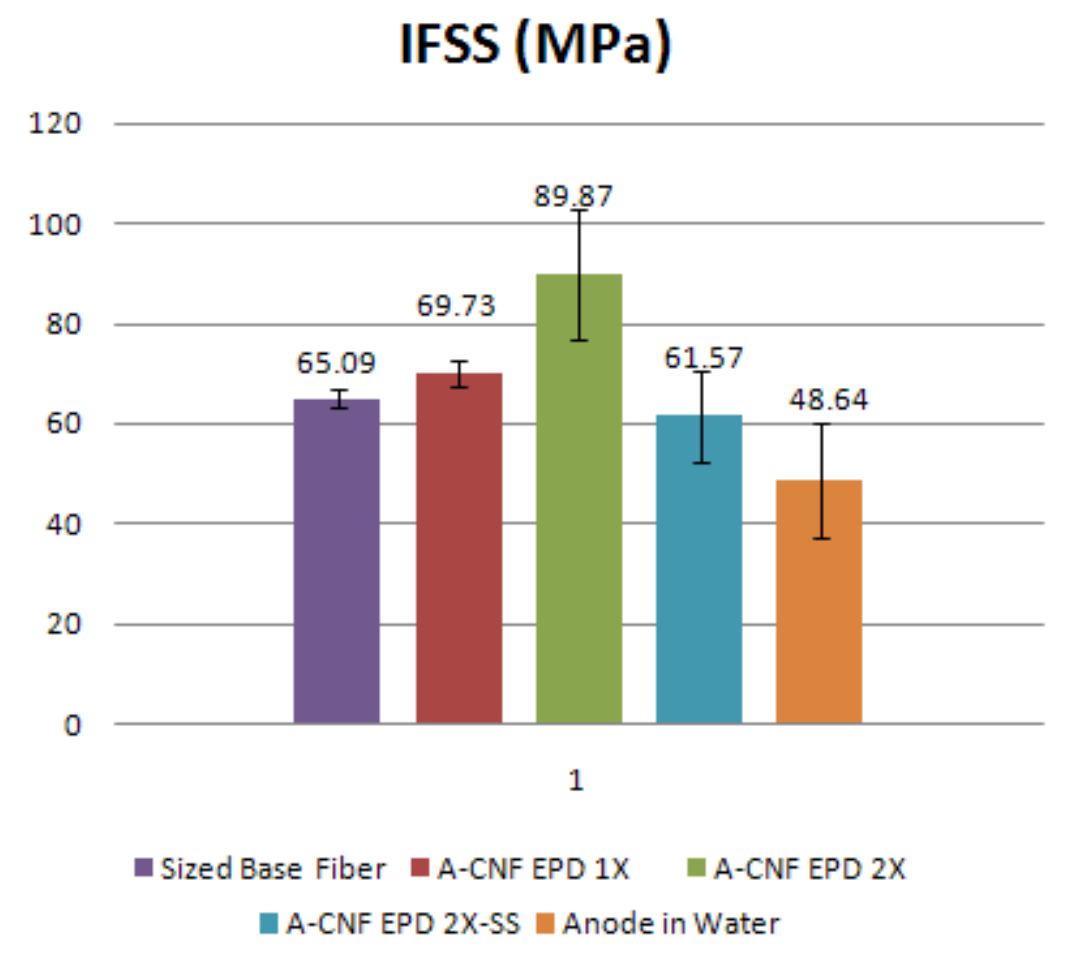

Figure 26: IFSS for one-stage fibers. 
Presented in Figure 26, this agglomerate-covered interface provided an IFSS increase of $38.2 \%$ over the base value. The 'sequential sonication' approach removed the presence of agglomerates and created an interface that appeared rough under the microscope (Figure 25C). While the agglomerates were reduced, the IFSS for the 2X-SS samples was $61.46 \mathrm{MPa}$, approximately $-5.35 \%$ less than the base value. Additionally, fibers acting as the anode in water for 1 min produced a decrease of $-25.22 \%$. Based on the stiffness concept presented by Thostenson et al. [9], this decrease in IFSS may be due to a decrease in interface stiffness. It appears that the above A-CNF deposition does little to enhance the interface, and at best helps to maintain the interface stiffness and prevent the overall stress transfer from being significantly lowered. The 1X A-CNF deposited interface had an IFSS that is $26.58 \%$ higher than the anode fibers in water. Doubling the concentration and sequentially mixing the A-CNF solution led to an IFSS increase of $43.94 \%$ over the anode fiber value, while the agglomerate covered surface resulting from non-sequential mixing had an increase of 84.79\%. Increasing A-CNF deposition on the anode fiber surface appears to restrict stiffness loss at the interface which prevents stress transfer from being lowered. In comparison, fibers acting as the cathode for 1 min of O-CNF deposition (Figure 27) produced an IFSS value of 204.8 MPa, which is an 215\% increase over the base fiber samples and close to the increase of $207 \%$ previously reported [81]. Also in the previous investigation, it was shown that sized fibers acting as the cathode in water without OCNFs produced a rough interface with degraded sizing; this resulted in an IFSS increase of 79\%.

Recently, Lee et al. [8] performed a one-stage EPD process to deposit CNFs and MWCNTs onto carbon fabric layers. Of particular note, they reported a $13 \%$ increase in interlaminar shear strength (ILSS) for composites made using fabrics acting as the cathode during a co-deposition of carboxylic acid functionalized MWCNTs and copper nanoparticles was 
reported. Additionally, they reported less homogenous deposition for nanoparticles on fabric acting as the anode. Compared to the base panel, a 10\% decrease in ILSS was reported for composites made from CNF deposited layers acting as the anode, while only a slight increase was reported for composites made from CNF-deposited layers acting as the cathode. In the approach presented by Rodriguez, et al. [7], composites made from a single deposition stage of O-CNFs onto carbon fabric layers resulted in an ILSS increase of 9\%; the fabric layers acted as the cathode. Furthermore, an ILSS increase of 10\% was achieved for composites composed of A-CNF deposited carbon fabric layers using a two-stage process in which the layers acted as first the cathode and then the anode.

In order to further investigate the two-stage methodology, single fiber experiments were conducted for fibers treated using the two-stage process, wherein, the fibers first acted as the cathode and then acted as the anode. As indicated previously in Table 4, the Weibull slope and scale parameters for these two-stage process, sequentially sonicated A-CNF deposited fibers (ACNF EPD 2S-2X-SS) are similar to those fibers acting as the cathode for O-CNF deposition. This indicates that the additional minute of exposure to the anodic electric field did not further enhance or degrade the fiber tensile strength. Figure 28 compares the polarized optical microscope images for this fiber type and the fibers acting as the anode in ultrapure water. The two-stage A-CNF deposited fibers (A) show breaks with adjacent birefringes which indicate quick stress transfer between matrix and fiber. In Figure 28B, the birefringes extend far along the fiber surface, indicating little interaction between the fiber and the matrix. 


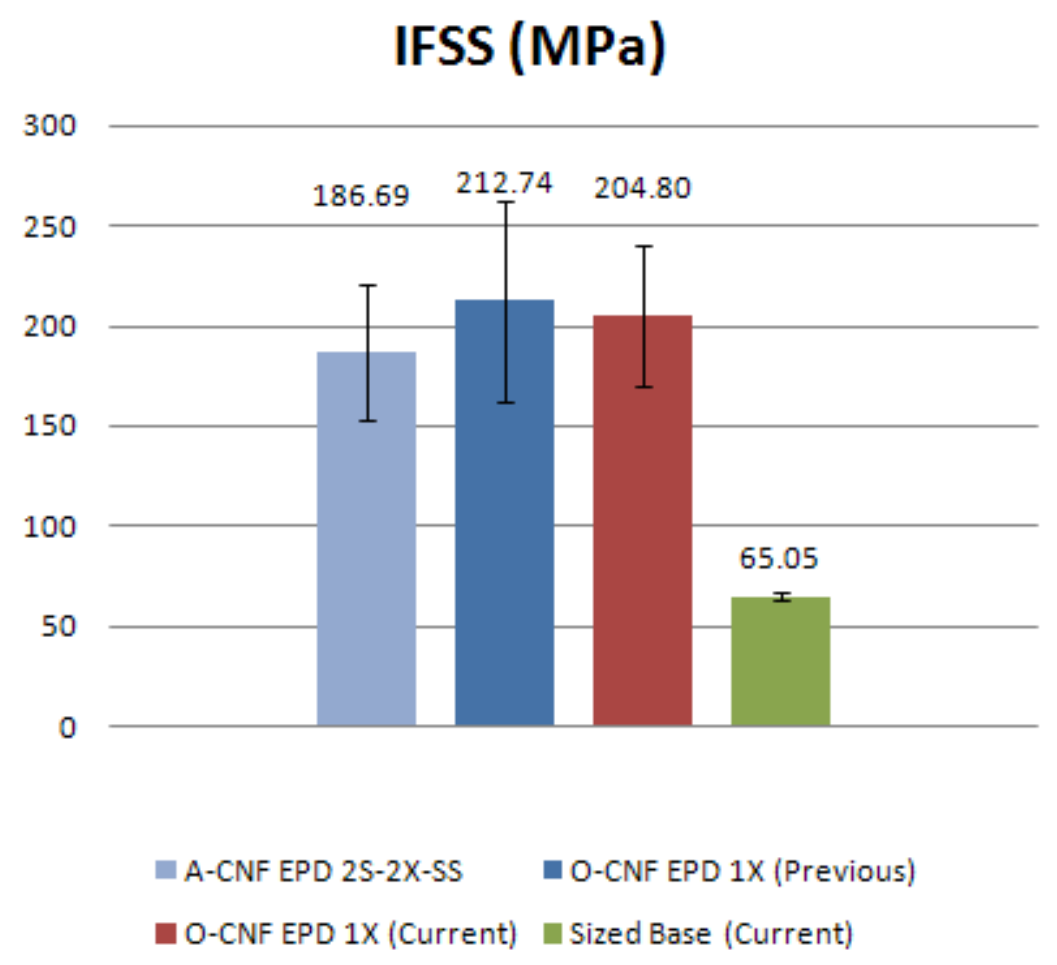

Figure 27: Two-stage A-CNF and one-stage O-CNF comparison.

As depicted in Figure 27, the two-stage A-CNF deposited samples provided an IFSS of 186.69 MPa, which is an $~ 187 \%$ increase compared to the base fiber. Also included in the figure are the IFSS values for O-CNF deposited fibers from the previous and current works. Combined with the investigations of Lee et al. [8] and Rodriguez et al. [7], it is clear that fibers acting as the cathode play a critical role in enhancing the deposition of O-CNFs and A-CNFs which affects composite properties at both the single fiber and laminate levels. It also signifies the possibility that the two-stage approach affects A-CNFs in a manner that causes them to better reinforce the interface than the one-stage process. 


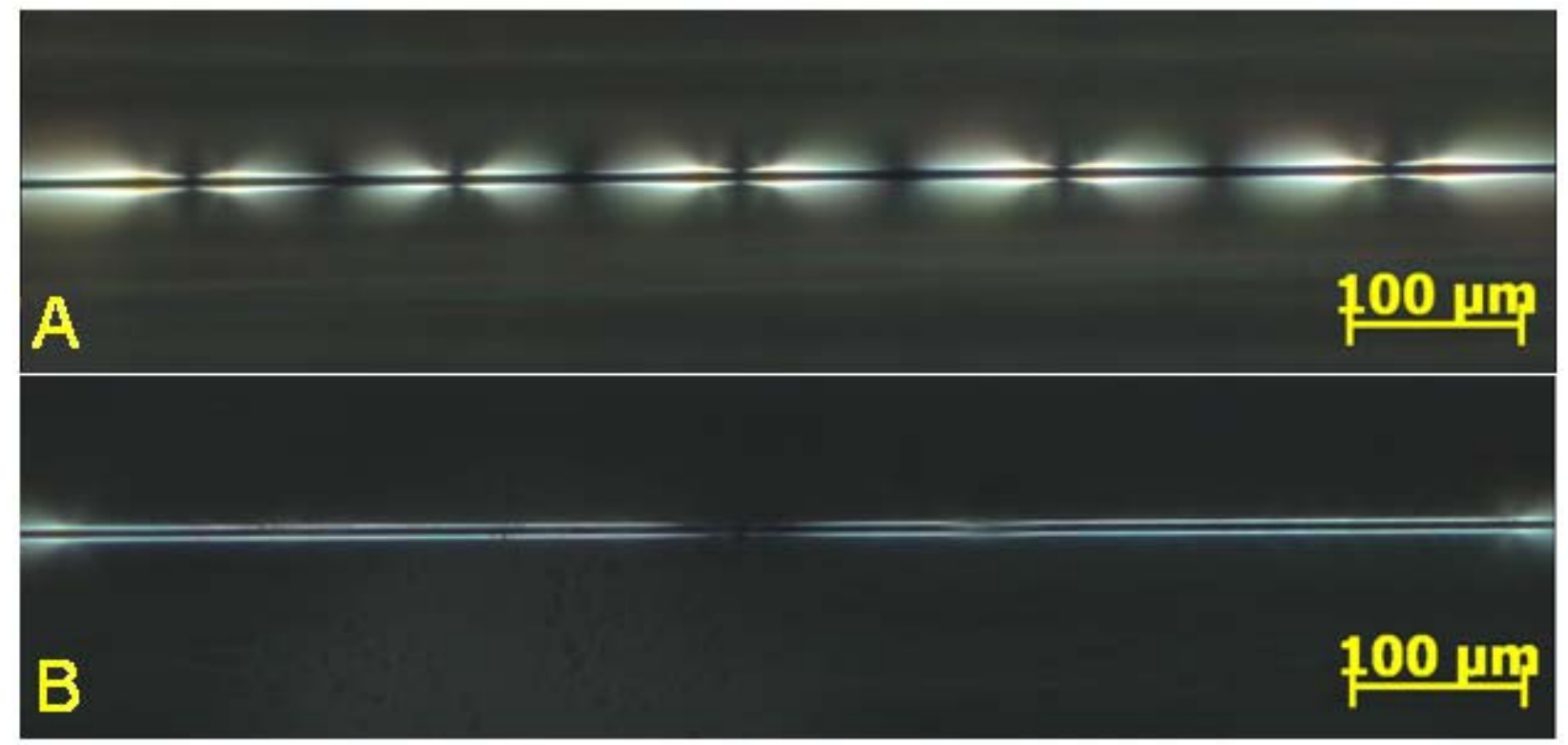

Figure 28: Polarized light birefringes for two-stage A-CNF deposition fibers (A) and fibers acting as the anode in water $(\mathrm{B})$.

\subsubsection{Fiber Surface Characterization}

In Figure 29, the number of breaks per incremental stress level for the fragmentation test is presented. The base fiber samples produced a uniform response to increasing stress, with saturation occurring primarily between 60 and $70 \mathrm{MPa}$. The low variability in the number of fiber breaks at saturation appears to indicate uniform stress transfer along a homogenous interface. Fibers acting as the anode provided a more variable break response to increasing stress, which may indicate a less homogenous interface. Fibers undergoing 1 min of $1 \mathrm{X}$ A-CNF deposition presented a uniform break response, while increasing to the $2 \mathrm{X}$ amount resulted in increased saturation variation for both the sequential and non-sequential sonication methods. The O-CNF and two-stage A-CNF deposition samples also presented a large variation in the final number of breaks at saturation, however, the values were much higher than the one-stage A-CNF samples. Also noticeable is that these samples reached saturation at a generally lower stress range of 50-60 MPa, which appears to indicate enhanced interface stress transfer. 


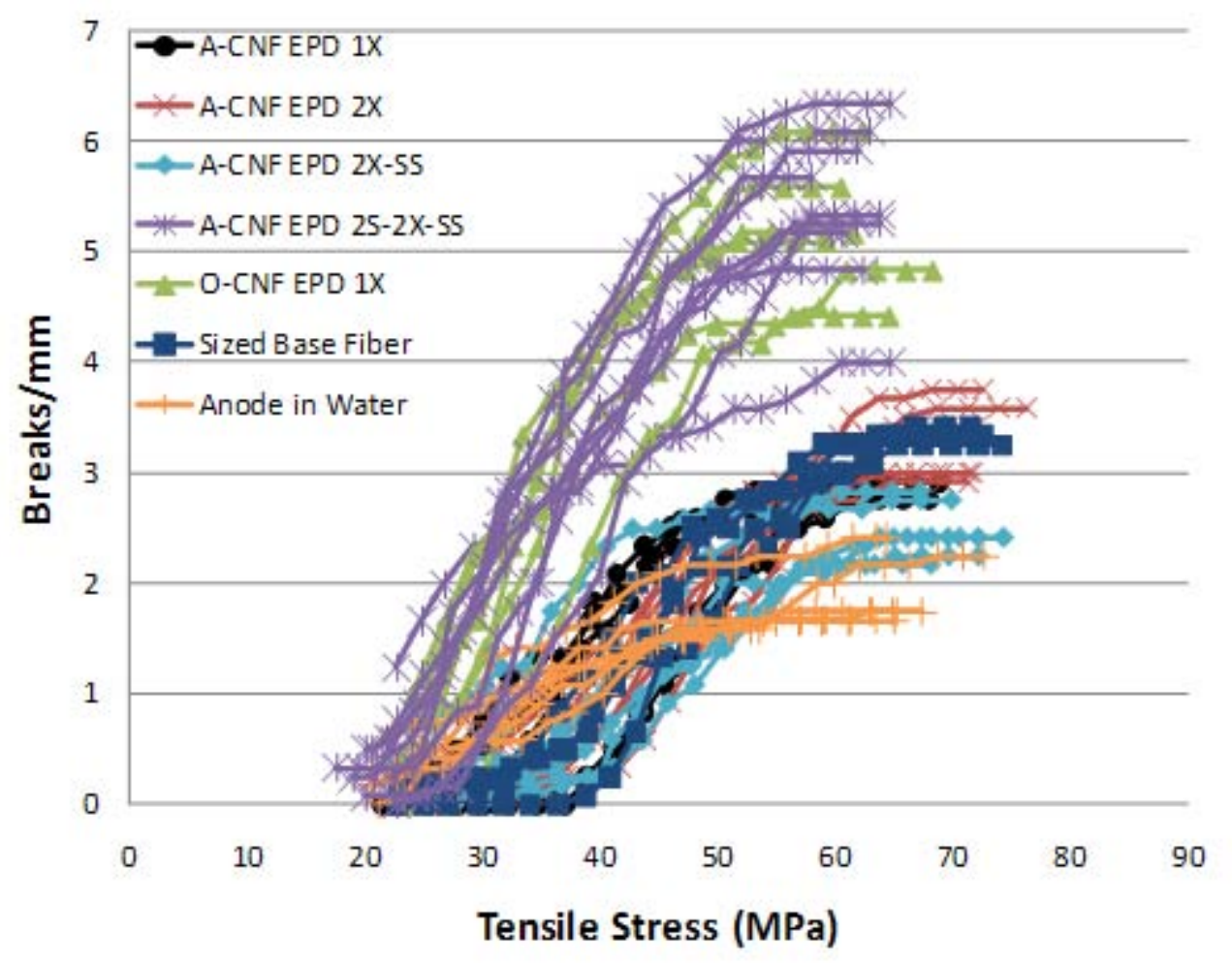

Figure 29: Break response under increasing applied tensile stress.

A histogram of the tensile failures for fibers acting as the anode in water, cathode in water, and during two-stage A-CNF deposition is provided to compare the relative failure densities for each treatment. In Figure 30, the base fibers failed most often between 3-5 GPa. 


\section{Tensile Failure Frequency}

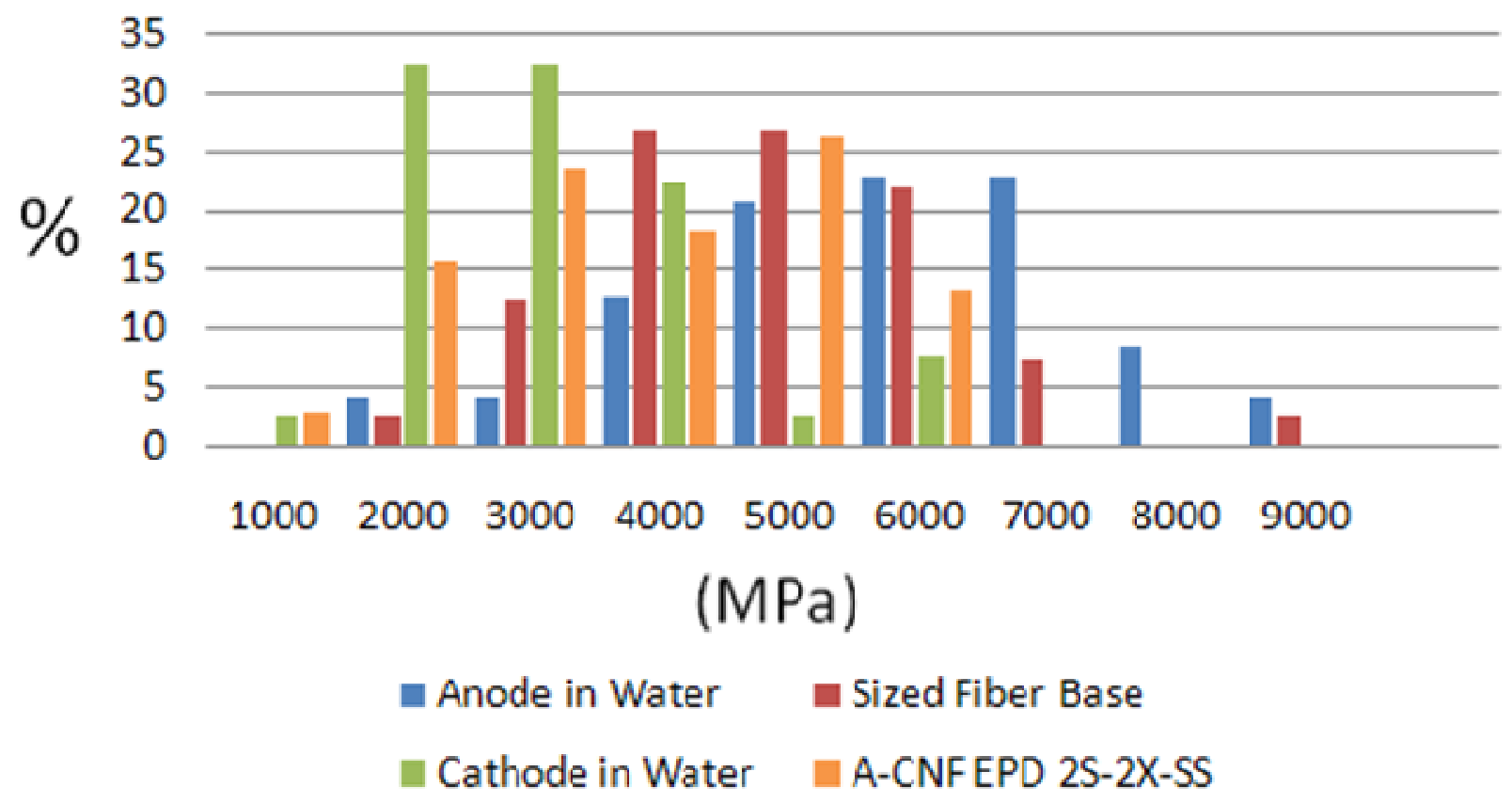

Figure 30: Frequency of tensile failure strength for base sized fibers, sized fibers acting as the anode, and sized fibers acting as the cathode. Field applied in water without presence of OCNFs or A-CNFs. Cathode data from Schaefer et al. [81].

For those fibers acting as the anode in water, there is a noteworthy shift to the right, with fibers having the most breaks in the 5 to $7 \mathrm{GPa}$ range. Also included is a histogram of the fibers acting as the cathode for $1 \mathrm{~min}$ in the previous study. The base average tensile strength of these fibers was originally found to be $4517 \mathrm{MPa}$, which is similar to the base value of $4429 \mathrm{MPa}$ determined for the base fibers used in this study. Accordingly, the shift to lower failure strength is apparent with a majority of failures occurring between 1 and $3 \mathrm{GPa}$. Interestingly, the fibers acting as both anode and cathode during the two-stage A-CNF deposition exhibit similar failure densities as the respective one-stage treatments. These results may indicate the development of different failure modes due to alteration of the carbon fiber interior and/or surface. 
Fragmentation samples were failed in tension and sputter coated with gold for imaging of the failure surface on an FESEM. Due to the inherent non-conductivity of the polymer matrix, significant charging of the failure surface occurred. In the upper left and right images of Figure 31, the pull-out surface for two different O-CNF deposited fibers is shown.

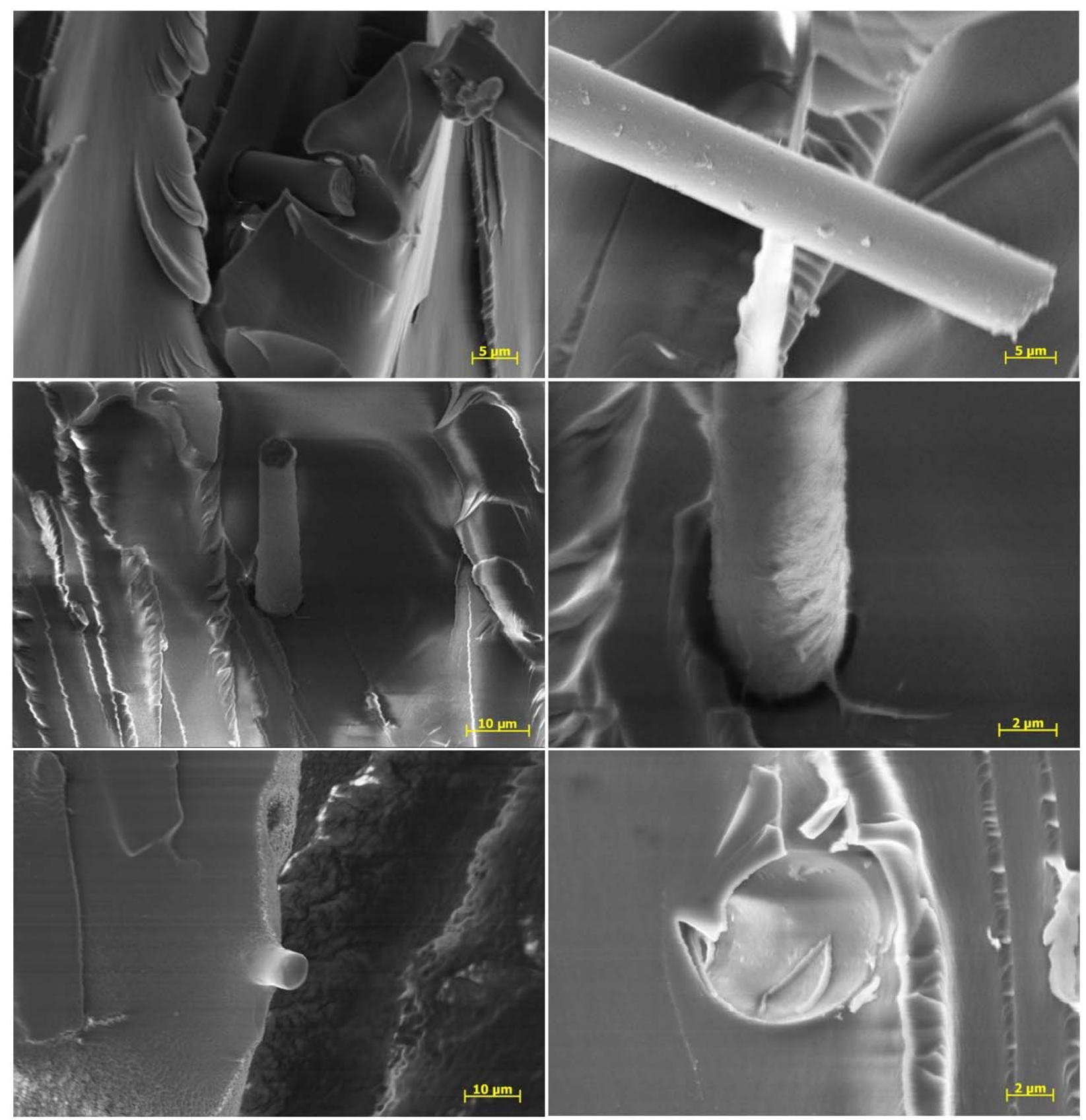

Figure 31: Comparison of tensile failure samples for samples undergoing O-CNF EPD (top), 2S2X-SS EPD (middle), and anode exposure in water (bottom). 
In the left image, the fiber surface appears smooth and the resin is delaminated from the fiber base. In the right image, the fiber surface appears to be covered with small pieces of resin or sizing. A 2S-2X- SS fiber is shown protruding from the failure surface in the middle left image, and a magnified view is shown in the middle right image. The fiber surface has increased roughness compared to the O-CNF deposited fiber, and A-CNF can be seen extending from the fiber surface into the matrix. This indicates the CNFs are effectively bridging the interface to enhance stress transfer.

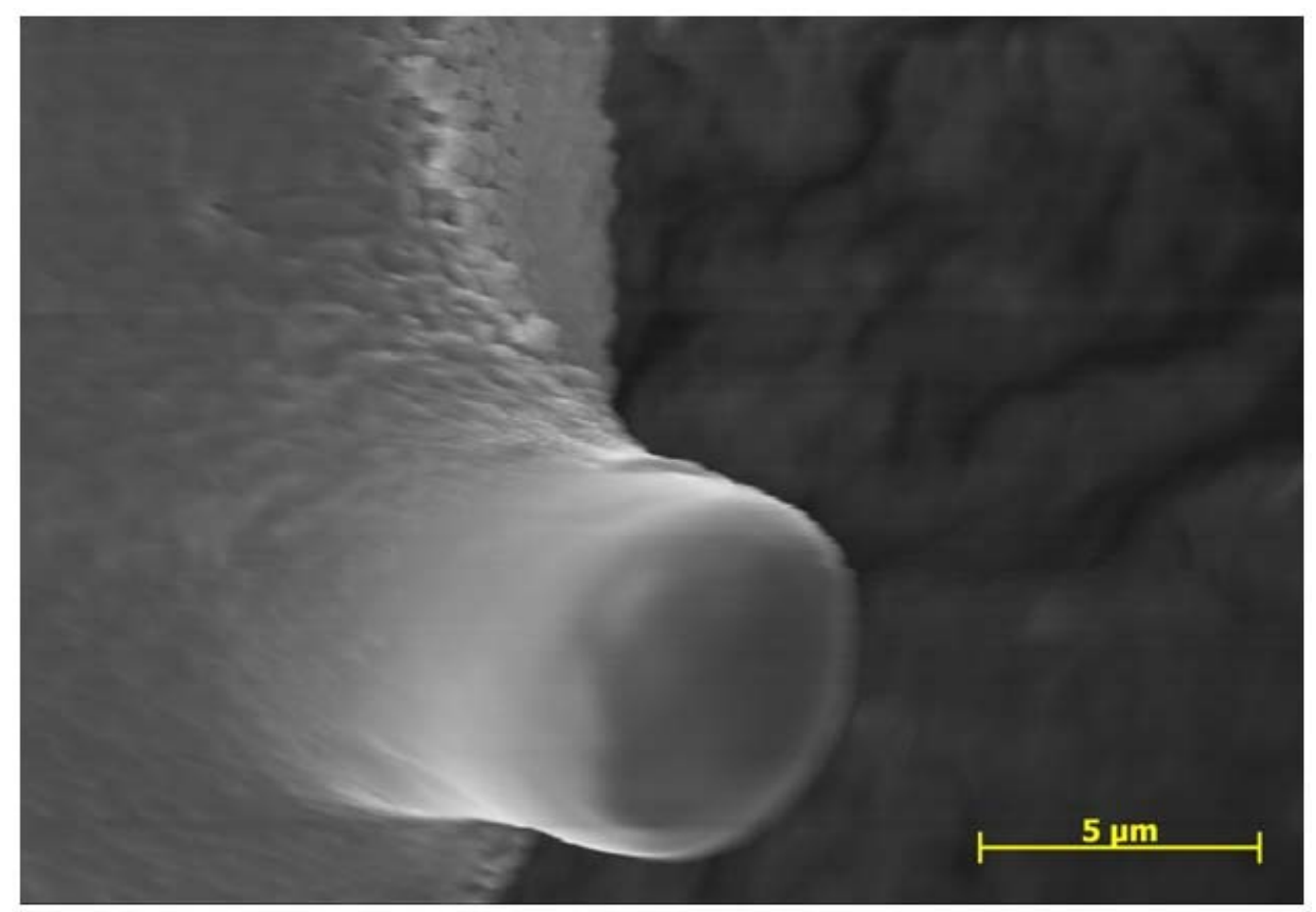

Figure 32: Magnified view of the anode fiber fragmentation failure surface.

The fragmentation failure surface for fibers acting as the anode in water without nanoparticles is shown in the bottom left of Figure 31 and a close up view is shown in Figure 32. An interesting difference between this fiber and the others shown is that the matrix appears to be closely adhered to the fiber and there is no apparent delamination. It appears that the interaction between the constituents would be enhanced, however, the IFSS is actually lower than the base 
sample level. It is proposed that instead of enhanced stiffness interaction, the matrix, sizing, and fiber interface has instead increased in toughness which has allowed for the absorbing of energy during fracture and prevented the delamination present in the other samples. This potential toughness increase would help prevent debonding under the tensile failure and allow for some flexibility between the fiber and matrix; thus, cause a decrease in stress transfer between the bulk matrix and embedded carbon fiber. In the bottom right image of Figure 31 the pullout location for the fiber in the left image is shown. Inside the hole, what appears to be sizing can be seen which further indicates the sizing may be interacting more closely with the matrix than the carbon fiber surface. In this case, the failure location would be at the fiber-sizing interface. 


\section{CHAPTER 5}

\section{CONCLUSIONS AND RECOMMENDATIONS}

\subsection{Conclusions}

The effects of electrophoretically deposited functionalized CNFs on the single carbon fiber-epoxy matrix interface were characterized. The aim of the research was to develop a fundamental understanding of the influence of functionalized CNF addition, EPD methodology, and fiber sizing on the IFSS and fiber surface morphology. Additionally, the processing effects on single fiber tensile strength were determined.

Single fibers coated with O-CNFs and A-CNFs in one-stage and two-stage deposition cycles were tested for IFSS using the fragmentation technique in conjunction with Weibull analysis of single fiber tensile failures. Sized fibers were tested to provide a base for comparing all samples, and it was shown that removing the sizing decreased the IFSS by $27 \%$. The onestage addition of O-CNFs to the unsized interface increased the IFSS by $15 \%$ over the base sized fiber and 56\% compared to the unsized fibers. One-stage cathodic deposition of O-CNFs on the single fiber surface led to an IFSS increases of more than 200\%. The IFSS increase was attributed to an enhancement in surface roughness and formation of additional surface area created by a homogeneous deposition of closely bound short and long O-CNFs that were axially aligned with the carbon fiber. One-stage A-CNF deposition was performed using two different mixing and concentration methodologies. The greatest increase in IFSS occurred for samples coated with layered and agglomerated CNFs. This 38.2\% IFSS increase was attributed to an increased stiffness of the material at the interface due to the presence of additional A-CNFs on the fiber surface. A further IFSS increase was produced by subjecting the fiber to a two-stage deposition approach with the fibers acting first as the cathode and then the anode. This approach resulted in an IFSS increase of $187 \%$ over the base value. 
While the IFSS decreased by approximately $25 \%$ for sized fibers acting as the anode in ultrapure water, sized fibers acting as the cathode in ultrapure water created a rough fiber surface with degraded sizing; accordingly, the IFSS increased by 79\%. However, the tensile strength of these cathode fibers was significantly reduced while the anode fibers maintained their strength. Interestingly, unsized fibers did not appear to be significantly affected by exposure to the cathodic DC electric field.

Based on these results, it appears that using EPD to place functionalized CNFs on the surface of single carbon fibers is an effective way to enhance the fiber-matrix interface. The IFSS increase was similar for the one-stage EPD of O-CNFs and the two-stage EPD of A-CNFs. The IFSS enhancement appears to be due to a combination of sizing degradation and deposited functionalized CNFs. The cathode stage of deposition appears to have a deleterious effect on sized fiber tensile strength. Fiber surfaces were examined using an FESEM, and the morphology indicated surface degradation and surface roughness for samples with the highest IFSS values. Further investigation of the difference between O-CNF and A-CNF interface reinforcement could provide important insight; however, it is clear that the presence of sizing has an impact on the tensile and interface properties of single carbon fibers acting as either the cathode or anode.

It is important to note that both the base sized fibers and the sized fibers deposited with O-CNFs were tested twice and that the resulting IFSS values were similar for each of the two samples. This validates the repeatability of the technical approach. With these results, it is intended that the methodologies for using EPD to place nanoparticles at the fiber-matrix interface may be improved to produce composite materials with superior stiffness, strength, and decreased weight. 


\subsection{Recommendations for Further Studies}

Based on the conclusions, the following investigations may provide additional insight:

- Determine the tensile modulus of the fibers. This would provide additional understanding of how the electric field affects fiber mechanical properties.

- $\quad$ Perform further failure analysis of fragmentation specimens using an optical microscope and FESEM to characterize the failure mode and location of fiber breaks. The goal would be to compare and contrast O-CNF and A-CNF reinforced interfaces to better understand the reinforcement mechanism and assess potential chemical bonding.

- The tensile strength of carbon fibers coated with sizing is affected by a cathodic DC electric field. By testing fibers from larger scale fiber collections (tow/panel) the impact of damage could be determined across a larger scale and the correlation between single fibers and composite laminates would be addressed. This investigation would determine the effect of current density on single fibers and would evaluate how changing the DC electric field strength affects fiber sizing and surface properties. This analysis would increase the understanding of EPD scalability, and may result in additional methods for interface enhancement.

- $\quad$ Perform similar work using functionalized SWCNTs or MWCNTs.

- Investigate the impact of solution concentration and conductivity on deposition amount and fiber mechanical properties.

- $\quad$ Process enhancement of the two-stage EPD approach. 
- $\quad$ Determine the tensile strength of unsized fibers acting as the anode. This may help determine why cathode based EPD damages single fibers and why anode based EPD does not. Performing surface chemistry analysis of both sample types may also indicate formation/deposition of any unknown groups or particles and indicate if sizing has been removed unknowingly. 
REFERENCES 


\section{LIST OF REFERENCES}

1. Herrera-Franco P, Drzal L.T. Comparison of Methods for the Measurement of Fibre/Matrix Adhesion in Composites. Composites. 1992; 23(1):2-27.

2. Ramanathan T., Bismarck A., Schulz E, Subramanian K. Investigation of the influence of acidic and basic surface groups on carbon fibres on the interfacial shear strength in an epoxy matrix by means of single-fibre pull-out test. Composites Science and Technology. 2001; 61:599-605.

3. Hughes J.D.H. The carbon fibre/epoxy interface--A review. Composites Science and Technology. 1991; 41(1):13-45.

4. Zhang Q., Liu J., Sager R., Dai L., Baur J. Hierarchical composites of carbon nanotubes on carbon fiber: Influence of growth condition on fiber tensile properties. Composites Science and Technology. 2009; 69(5):594-601.

5. Qian H., Bismarck A., Greenhalgh E.S., Shaffer M.S.P. Carbon nanotube grafted silica fibres: Characterising the interface at the single fibre level. Composites Science and Technology. 2010; 70(2):393-9.

6. Bekyarova E., Thostenson E.T., Yu A., Kim H., Gao J., Tang J., et al. Multiscale Carbon Nanotube-Carbon Fiber Reinforcement for Advanced Epoxy Composites. Langmuir. 2007; 23:3970-4.

7. Rodriguez A.J., Guzman M.E., Lim C-S., Minaie B. Mechanical properties of carbon nanofiber/fiber-reinforced hierarchical polymer composites manufactured with multiscale-reinforcement fabrics. Carbon. 2011; 49(3):937-48.

8. Lee S-B., Choi O., Lee W., Yi J-W., Kim B-S., Byun J-H., et al. Processing and Characterization of Multi-scale Hybrid Composites Reinforced with Nanoscale Carbon Reinforcements and Carbon Fibers. Composites Part A: Applied Science and Manufacturing.42(4):337-44.

9. Thostenson E.T., Li W.Z., Wang D.Z., Ren Z.F., Chou T-W. Carbon nanotube/carbon fiber hybrid multiscale composites. Journal of Applied Physics. 2002; 91(9):6034-7.

10. Zhang F-H., Wang R-G., He X-D., Wang C., Ren L-N. Interfacial shearing strength and reinforcing mechanisms of an epoxy composite reinforced using a carbon nanotube/carbon fiber hybrid. Journal of Materials Science. 2009; 44(13):3574-7.

11. Qian H., Bismarck A., Greenhalgh E.S., Kalinka G., Shaffer M.S.P. Hierarchical Composites Reinforced with Carbon Nanotube Grafted Fibers: The Potential Assessed at the Single Fiber Level. Chemical Materials. 2008; 20:1862-9. 


\section{LIST OF REFERENCES (continued)}

12. Zhandarov S., Mäder E. Characterization of fiber/matrix interface strength: applicability of different tests, approaches and parameters. Composites Science and Technology. 2005; 65(1):149-60.

13. Sager R.J., Klein P.J., Lagoudas D.C., Zhang Q., Liu J., Dai L. Effect of carbon nanotubes on the interfacial shear strength of T650 carbon fiber in an epoxy matrix. Composites Science and Technology. 2009; 69(7-8):898-904.

14. Palmeri M.J., Putz K.W., Ramanathan T., Brinson L.C. Multi-Scale Reinforcement of CFRPs using Carbon Nanofibers. Composites Science and Technology.71(2):79-86.

15. Boccaccini A.R., Cho J., Roether J.A., Thomas B.J.C., Jane Minay E., Shaffer M.S.P. Electrophoretic deposition of carbon nanotubes. Carbon. 2006; 44(15):3149-60.

16. Rodriguez A.J., Guzman M.E., Lim C-S., Minaie B. Synthesis of multiscale reinforcement fabric by electrophoretic deposition of amine-functionalized carbon nanofibers onto carbon fiber layers. Carbon. 2010; 48(11):3256-9.

17. Zhang J., Zhuang R., Liu J., Mäder E., Heinrich G., Gao S. Functional interphases with multi-walled carbon nanotubes in glass fibre/epoxy composites. Carbon. 2010; 48(8):2273-81.

18. Park J.K., Do I-H., Askeland P., Drzal L.T. Electrodeposition of exfoliated graphite nanoplatelets onto carbon fibers and properties of their epoxy composites. Composites Science and Technology. 2008; 68(7-8):1734-41.

19. Biest O.V.D., Put S., Anne G., Vleugels J. Electrophoretic deposition for coatings and free standing objects. Journal of Materials Science. 2004; 39:779-85.

20. Kelly A., Tyson W.R. Tensile properties of fibre-reinforced metals: copper/tungsten and copper/molybdenum. J Mech Phys Solids. 1965; 13:329-50.

21. Beyerlein I.J., Phoenix S.L. Statistics for the Strength and Size Effects of Microcomposites with Four Carbon Fibers in Epoxy Resin. Composites Science and Technology. 1996; 56:75-92.

22. Otani H., Phoenix S.L., Petrina P. Matrix Effects on Lifetime Statistics for Carbon FibreEpoxy Microcomposites in Creep Rupture. Journal of Materials Science. 1991; 26:195570. 


\section{LIST OF REFERENCES (continued)}

23. Hoecker F, Karger-Kocsis J. Effects of the interface on the mechanical response of CF/EP microcomposites and macrocomposites. Composites. 1994; 25(7):729-38.

24. Chau T.T. A review of techniques for measurement of contact angles and their applicability on mineral surfaces. Minerals Engineering. 2009; 22(3):213-9.

25. Milman N., Yoon J.K., Hickey A.J., Burgess D.J. Comparison of contact angle measurements by three methods. Colloids and Surfaces B: Biointerfaces. 1993; 1(5):31521.

26. Song B., Bismarck A., Tahhan R., Springer J. A Generalized Drop Length-Height Method for Determination of Contact Angle in Drop-on-Fiber Systems. Journal of Colloid and Interface Science. 1998; 197(1):68-77.

27. Rao V., Herrera-Franco P., Ozzello A.D., Drzal L.T. A direct comparison of the fragmentation test and the microbond pull-out test for determining the interfacial shear strength. Journal of Adhesion. 1991; 34:65-77.

28. Heilhecker H., Cross W., Pentland R., Griswold C., Kellar J.J., Kjerengtroen L. The Vice Angle in the Microbond Test. Journal of Materials Science Letters. 2000; 19(2145-2147).

29. Nishikawa M., Okabe T., Hemmi K., Takeda N. Micromechanical modeling of the microbond test to quantify the interfacial properties of fiber-reinforced composites. International Journal of Solids and Structures. 2008; 45(14-15):4098-113.

30. Paipetis A., Galiotis C., Ching Liu Y., Nairn J.A. Stress Transfer from the Matrix to the Fibre in a Fragmentation Test: Raman Experiments and Analytical Modeling. Journal of Composite Materials.1999; 33.

31. Wang X., Zhang B., Du S., Wu Y., Sun X. Numerical simulation of the fiber fragmentation process in single-fiber composites. Materials \& Design. 2010; 31(5):246470.

32. Bogoeva-Gaceva G., Mäder E., Häuler L., Sahre K. Parameters affecting the interface properties in carbon fibre/epoxy systems. Composites. 1995; 26(2):103-7.

33. Gaur U., Chou CT., Miller B. Effect of hydrothermal ageing on bond strength. Composites. 1994; 25(7):609-12.

34. Marshall P.I., Attwood D., Healey M.J. Interface chemistry in carbon fibre/epoxy matrix composites. Composites. 1994; 25(7):752-6. 


\section{LIST OF REFERENCES (continued)}

35. Yumitori S., Wang D., Jones F.R. The role of sizing resins in carbon fibre-reinforced polyethersulfone (PES). Composites. 1994; 25(7):698-705.

36. $\quad$ Cheng T.H., Zhang J., Yumitori S., Jones F.R., Anderson C.W. Sizing resin structure and interphase formation in carbon fibre composites. Composites. 1994; 25(7):661-70.

37. Buxton A., Baillie C. A study of the influence of the environment on the measurement of interfacial properties of carbon fibre/epoxy resin composites. Composites. 1994; 25(7):604-8.

38. Paipetis A., Galiotis C. Effect of fibre sizing on the stress transfer efficiency in carbon/epoxy model composites. Composites Part A: Applied Science and Manufacturing. 1996; 27(9):755-67.

39. Wimolkiatisak A.S., Bell J.P. Interfacial Shear Strength and Failure Modes of InterphaseModified Graphite-Epoxy Composites. Polymer Composites. 1989; 10(3):162-72.

40. Hamada H., Ikuta N., Maekawa Z., Ichihashi H., Nishida N. Evaluation of the i nterfacial properties in the embedded-single-fibre coupon test. Composites Science and Technology. 1993; 48(1-4):81-5.

41. Iijima S. Helical microtubules of graphitic carbon. Nature. [10.1038/354056a0]. 1991; 354(6348):56-8.

42. Iijima S., Ichihashi T. Single-shell carbon nanotubes of 1-nm diameter. Nature. [10.1038/363603a0]. 1993; 363(6430):603-5.

43. Bethune D.S., Klang C.H., de Vries M.S., Gorman G., Savoy R., Vazquez J., Cobaltcatalysed growth of carbon nanotubes with single-atomic-layer walls. Nature. [10.1038/363605a0]. 1993; 363(6430):605-7.

44. Monthioux M., Kuznetsov V.L. Who should be given the credit for the discovery of carbon nanotubes? Carbon. 2006; 44(9):1621-3.

45. Terrones M. Science and Technology of the Twenty First Century: Synthesis, Properties, and Applications of Carbon Nanotubes. Annual Review of Materials Research. 2003; 33:419-501.

46. Oberlin A., Endo M., Koyama T. Filamentous growth of carbon through benzene decomposition. Journal of Crystal Growth. 1976; 32(3):335-49.

47. Al-Saleh M.H., Sundararaj U. A review of vapor grown carbon nanofiber/polymer conductive composites. Carbon. 2009; 47(1):2-22. 


\section{LIST OF REFERENCES (continued)}

48. Tibbetts G.G., Lake M.L., Strong K.L., Rice B.P. A review of the fabrication and properties of vapor-grown carbon nanofiber/polymer composites. Composites Science and Technology. 2007; 67(7-8):1709-18.

49. Ozkan T., Naraghi M., Chasiotis I. Mechanical properties of vapor grown carbon nanofibers. Carbon. 2010; 48(1):239-44.

50. Lakshminarayanan P.V., Toghiani H., Pittman Jr C.U. Nitric acid oxidation of vapor grown carbon nanofibers. Carbon. 2004; 42(12-13):2433-42.

51. Finegan I.C., Tibbetts G.G., Glasgow D.G., Ting J-M., Lake M.L. Surface treatments for improving the mechanical properties of carbon nanofiber/thermoplastic composites. Journal of Materials Science. 2003; 38(16):3485-90.

52. Soo-Na A., Hwa-Jeong L., Bang-Jun K., Loon-Seng T., Jong-Beom B. Epoxy/AmineFunctionalized Short-Length Vapor-Grown Carbon Nanofiber Composites. Journal of Polymer Science Part A: Polymer Chemistry. 2008; 46(22).

53. Prolongo S.G., Campo M., Gude M.R., Chaos-Morán R., Ureña A. Thermo-physical characterisation of epoxy resin reinforced by amino-functionalized carbon nanofibers. Composites Science and Technology. 2009; 69(3-4):349-57.

54. Tibbetts G.G., Kway C., Howe J.Y., Lake M.L. Heat Treating Carbon Nanofibers for Optimal Composite Performance. 48th International SAMPE Symposium. 2003; 48:213645.

55. www.cheaptubes.com/carbon-nanotubes-prices.htm. Retrieved 2010.

56. Pyrograf Products I. 'CNF and CNT Comparison.' Retrieved 2010.

57. Moniruzzaman M., Winey K.I. Polymer Nanocomposites Containing Carbon Nanotubes. Macromolecules. [Review]. 2006; 39:5194-205.

58. Song Y.S., Youn J.R. Influence of dispersion states of carbon nanotubes on physical properties of epoxy nanocomposites. Carbon. 2005; 43(7):1378-85.

59. Gong X., Liu J., Baskaran S., Voise R.D., Young JS. Surfactant-Assisted Processing of Carbon Nanotube/Polymer Composites. Chemical Materials. 2000; 12:1049-52.

60. Pan Y., Li L., Chan S.H., Zhao J. Correlation between dispersion state and electrical conductivity of MWCNTs/PP composites prepared by melt blending. Composites Part A: Applied Science and Manufacturing. 2010; 41(3):419-26. 


\section{LIST OF REFERENCES (continued)}

61. Barick A.K., Tripathy D.K. Effect of nanofiber on material properties of vapor-grown carbon nanofiber reinforced thermoplastic polyurethane (TPU/CNF) nanocomposites prepared by melt compounding. Composites Part A: Applied Science and Manufacturing. 2010; 41(10):1471-82.

62. Zhu J., Kim J., Peng H., Margrave J.L., Khabashesku V.N., Barrera E.V. Improving the Dispersion and Integration of Single-Walled Carbon Nanotubes in Epoxy Composites through Functionalization. Nano Letters. [Letter]. 2003 April 21, 2003; 3(8):1107-13.

63. Gojny F.H., Nastalczyk J., Roslaniec Z., Schulte K. Surface modified multi-walled carbon nanotubes in CNT/epoxy-composites. Chemical Physics Letters. 2003; 370(56):820-4.

64. Garcia E.J., Wardle B.L., John Hart A., Yamamoto N. Fabrication and multifunctional properties of a hybrid laminate with aligned carbon nanotubes grown In Situ. Composites Science and Technology. 2008; 68(9):2034-41.

65. Besra L., Liu M. A review on fundamentals and applications of electrophoretic deposition (EPD). Progress in Materials Science. 2007; 52(1):1-61.

66. Yang Y.J., Zhao G.J., Hu S. Direct current electrodeposition of carbon nanofibers in DMF. Electrochemistry Communications. 2007; 9(11):2681-5.

67. Rigueur J.L., Hasan S.A., Mahajan S.V., Dickerson J.H. Buckypaper fabrication by liberation of electrophoretically deposited carbon nanotubes. Carbon.In Press, Accepted Manuscript.

68. Song Y.S. Multiscale Fiber-Reinforced Composites Prepared by Vacuum-Assisted Resin Transfer Molding. Polymer Composites. [Online Article]. 2007.

69. Moisala A., Li Q., Kinloch I.A., Windle A.H. Thermal and electrical conductivity of single- and multi-walled carbon nanotube-epoxy composites. Composites Science and Technology. 2006; 66(10):1285-8.

70. Gou J., Minaie B, Wang B., Liang Z., Zhang C. Computational and experimental study of interfacial bonding of single-walled nanotube reinforced composites. Computational Materials Science. 2004; 31(3-4):225-36.

71. Yang S-Y., Lin W-N., Huang Y-L., Tien H-W., Wang J-Y., Ma C-CM. Synergetic effects of graphene platelets and carbon nanotubes on the mechanical and thermal properties of epoxy composites. Carbon. 2010; In Press. Accepted Manuscript. 


\section{LIST OF REFERENCES (continued)}

72. Lafdi K., Matzek M. Carbon nanofibers as a nano-reinforcement for polymeric nanocomposites. In: 48th International SAMPE symposium proceedings, Long Beach, USA. 2003.

73. Choi Y-K., Sugimoto K-i., Song S-M., Gotoh Y., Ohkoshi Y., Endo M. Mechanical and physical properties of epoxy composites reinforced by vapor grown carbon nanofibers. Carbon. 2005; 43(10):2199-208.

74. Yang Y., Gupta M.C., Dudley K.L., Lawrence R.W. The fabrication and electrical properties of carbon nanofibre-polystyrene composites. Nanotechnology. 2004;14:15458.

75. Green K.J., Dean D.R., Vaidya U.K., Nyairo E. Multiscale fiber reinforced composites based on a carbon nanofiber/epoxy nanophased polymer matrix: Synthesis, mechanical, and thermomechanical behavior. Composites Part A: Applied Science and Manufacturing. 2009; 40(9):1470-5.

76. Rodriguez A., Guzman M., Lim C., Minaie B. Electrophoretic deposition of CNF onto carbon fiber layers for manufacturing of fiber-reinforced polymer nanocomposites. SAMPE '09 Spring Conference Proceedings. 2009 May 18, 2009 - May 21, 2009.

77. Rodriguez A., Guzman M., Lim C., Minaie B. Hybrid Carbon Nanofiber-Carbon Fiber Layers For Manufacturing of Fiber-Reinforced Polymer Nanocomposites. SAMPE '09 Spring Conference Proceedings. 2010 May 17, 2010 - May 20, 2010.

78. Lim CS. Mechanical and Electrical Properties of Aligned Carbon Nanofiber/Epoxy Nanocomposites. Masters Thesis, Wichita State University. 2010.

79. Rodriguez A.J. Processing and Characterization of Multiscale-Reinforced Polymer Composites. Wichita State University Doctoral Thesis. 2010.

80. Watson A.S., Smith R.L. An Examination of Statistical Theories for Fibrous Materials in the Light of Experimental Data. Journal of Materials Science. 1985; 20:3260-70.

81. Schaefer J.D., Rodriguez A.J., Guzman M.E., Lim C-S., Minaie B. Effects of Electrophoretically Deposited Carbon Nanofibers on the Interface of Single Carbon Fibers Embedded in Epoxy Matrix. Carbon. 2011; 49(8): 2750-2759.

82. Umesh G., Bernard M. Microbond Method for Determination of the Shear Strength of a Fiber/Resin Interface: Evaluation of Experimental Parameters. Composites Science and Technology. 1989; 34:35-51. 